\title{
New fabrication method for producing reduced graphene oxide flexible electrodes by using low-power visible laser diode engraving system
}

\author{
A. Chuquitarqui, ${ }^{a}$ L.C. Cotet, ${ }^{\text {b,c* }}$ M. Baia, ${ }^{d}$ E. György, ${ }^{\text {a,e }}$ K. Magyari, ${ }^{\text {f,g }}$ L. Barbu- \\ Tudoran, ${ }^{\text {h,i }}$ L. Baia, ${ }^{\mathrm{c}, \mathrm{d}}$ M. Díaz-González, ${ }^{\mathrm{j}}$ C. Fernández-Sánchez, ${ }^{\mathrm{j}}$ A. Pérez del Pino ${ }^{\mathrm{a},{ }^{*}}$
}

a. Instituto de Ciencia de Materiales de Barcelona, Consejo Superior de Investigaciones Científicas (ICMAB-CSIC), Campus UAB, 08193 Bellaterra, Spain

b. Faculty of Chemistry and Chemical Engineering, Babes-Bolyai University, Department of Chemical Engineering, Arany Janos str.11, RO-400028, Cluj-Napoca, Romania.

c. Institute of Research-Development-Innovation in Applied Natural Sciences, BabesBolyai University, Fântânele str. 30, RO-400294, Cluj-Napoca, Romania

d. Faculty of Physics, Babes-Bolyai University,M. Kogalniceanu str. 1, RO-400048, ClujNapoca, Romania

e. National Institute for Lasers, Plasma and Radiation Physics, PO Box MG 36, 77125

Bucharest, Romania

f. Institute of Interdisciplinary Research in Bio-Nano-Science, Babes-Bolyai University Treboniu Laurian 42, RO-400271, Romania

g. Institute of Chemistry, University of Szeged, H-6720 Szeged, Tisza Lajos krt. 103, Hungary

h. Electron Microscopy Laboratory "Prof. C. Craciun", Faculty of Biology \& Geology, Babes-Bolyai University, 5-7 Clinicilor Str., 400006, Cluj-Napoca

i. Electron Microscopy Integrated Laboratory, National Institute for R\&D of Isotopic and Molecular Technologies, 67-103 Donat Str., 400293 Cluj-Napoca, Romania j. Instituto de Microelectrónica de Barcelona (IMB-CNM), CSIC. Campus UAB, 08193 Bellaterra, Barcelona, Spain

Corresponding authors: L.C. Cotet: ccosmin@chem.ubbcluj.ro; A. Pérez del Pino: aperez@icmab.es, +34 935801853. 


\begin{abstract}
The fabrication of bendable electronic devices is being a scientific-technological area of very rapid advance in which new materials and fabrication techniques are being continuously developed. In this kind of devices, the fabrication of flexible conductive electrodes adherent to the substrate is a key factor. Besides, eco-friendliness, low cost and fast production are essential requirements for the successful progress of new technologies. In this work, a novel method for obtaining graphene-based flexible electrodes is presented. Conductive films were obtained by means of visible laser irradiation of graphene oxide layers deposited on polyethylene terephthalate substrates besides selfstanding membranes sandwiched between glass slides. Despite the low power of the laser system, numerical simulations indicate the development of temperatures over $1000 \mathrm{~K}$ throughout the irradiated material. The laser-induced spatially confined heating leads to the reduction of the graphene oxide material whereas the glass-based sandwich assembly avoids reoxidation from surrounding air. By scanning and pixelated modes, reduced graphene oxide electrodes, up to $100 \mu \mathrm{m}$ in thickness, and with resistivity as low as $6 \times 10^{-}$

${ }^{4} \Omega \mathrm{m}$ were obtained in an easy and versatile way. Proof-of-concept microsupercapacitors and electrochemical sensors were fabricated with this technique, showing promising performance.
\end{abstract}

Keywords: laser-reduced graphene oxide, flexible electrode, graphene electrode, laser fabrication.

\title{
1. Introduction
}

The advance of materials technology and the societal demand of flexible electronic devices is rapidly transforming the electronics industry. Different routes are being developed for the fabrication of bendable devices with increasing degree of sophistication in several application areas [1-3], being the methods based on solution-printing among the most studied ones [4-7]. Printing technologies exhibit full compatibility with bendable substrates, and their involvement also lead to the fabrication of patterns of functional materials by means of simple steps and low cost. Nevertheless, the performance of the obtained devices is still limited by the properties of the materials constituting the ink, and some technological issues such as those related to the transfer of unsuitable surface 
tension or viscous solutions are still challenging to be overtaken. As alternative, laserbased manufacturing methods of flexible electronics reveal unique advantages such as remarkable controllability, high precision and rapid processing times [8-10]. These techniques, based on fast local heating of the exposed material, allow inducing structural and compositional changes generally out of thermodynamic equilibrium besides controlled vaporization for patterning $[11,12]$.

The fabrication of soft conductive electrodes is a key factor in the development of bendable electronic devices, so great research efforts are being carried out in this particular issue. Inks composed of metallic nanoparticles, conductive polymers or graphene-based dispersions are being developed for this objective [13-16]. Particularly, and due to its outstanding electrical properties, striking advances are being obtained by using graphene as base material [17-19]. Nevertheless, certain challenges including the tendency of graphene sheets to aggregate in suspensions leading to inappropriate surface tension and viscosity, the absence of adhesion of the inks to the substrate and the lack of a cost-effective method for the synthesis of high quality graphene precursor, prevent the practical application of graphene in conductive inks. An interesting alternative route consists in the use of graphene oxide (GO) instead of graphene as precursor. GO sheets are obtained through the oxidation of graphite into graphite oxide, being subsequently exfoliated to individual sheets [20,21]. Afterwards, the GO sheets can be subjected to a reduction process for removing the oxygen-containing functional groups leading to the formation of reduced-graphene oxide (rGO) with graphene-like structure.

Generally, the reduction of GO can be carried out by means of different chemical, electrochemical or thermal procedures [22-26]. Depending on the system to be fabricated, chemical-electrochemical methods could exhibit materials incompatibility whereas the thermal treatments could provoke damage to temperature-sensitive substrates such as polymers. In this case, the use of a highly-controlled local thermal source as laser radiation ensures the production of rGO with negligible damage of the substrate or neighbouring sensitive materials [27]. Laser methods for the simultaneous reduction of GO, patterning and transfer to polymer substrates have been already published, though they are limited to thin layers [28, 29]. Recently, laser-induced reduction of GO is being intensively used for the development of devices in applications such as sensors, actuators, supercapacitors, solar cells and other electronic devices [30-43]. In these methods, different strategies are explored for avoiding the combustion of GO material. 
In this work, a novel route is shown for the easy and cost-effective fabrication of flexible graphene-based electrodes by means of laser processing. The method, which has been recently patented [44], consists in the low-power visible laser irradiation of GO layers sandwiched between glass slides for avoiding oxidation and burning of the irradiated GO material. In this way, laser-induced reduction of up to $100 \mu \mathrm{m}$ thick GO layers were both obtained on polyethylene terephthalate (PET) substrates and as free-standing membranes. The GO layers exhibit a substantial decrease of the sheet resistance after laser reduction process. Additionally, the promising performance of the resulting electrodes working as microsupercapacitor and electrochemical sensor devices, is shown.

\section{Experimental section}

\subsection{Sono-oxidative preparation of initial GO suspensions.}

The oxidative-exfoliation synthesis of GO material can be structured in three steps. In the first step, in an $800 \mathrm{~mL}$ glass beaker $270 \mathrm{ml}$ of $\mathrm{H}_{2} \mathrm{SO}_{4}$ (95-97\% purity, SC Nordic Invest SRL, Cluj-Napoca), $30 \mathrm{ml} \mathrm{H}_{3} \mathrm{PO}_{4}$ (85\% purity, Merck) and $2.7 \mathrm{~g}$ of graphite (powder, $\leq$ $0.1 \mathrm{~mm}$, Fulka) were mixed under stirring and placed in ice bath. When a T between 0 $5^{\circ} \mathrm{C}$ was achieved, $12 \mathrm{~g} \mathrm{KMnO}_{4}$ (99\%, Merck) was gradually added to the obtained mixture. The reaction beaker was sealed and kept in ice bath for $2 \mathrm{~h}$ and then without stirring at room temperature (RT) for 4 days. In the second step, $200 \mathrm{~mL}$ of $3 \% \mathrm{H}_{2} \mathrm{O}_{2}$ (SC “Hipocrate 2000” SRL, Bucharest) was slowly added to the previous mixture (in about $30 \mathrm{~min}$ ). The obtained slurry was centrifuged (5000 rpm/15 min), and the supernatant was removed. The remaining solid material was then washed successively with $200 \mathrm{~mL}$ of bidistilled $\mathrm{H}_{2} \mathrm{O}, 100 \mathrm{~mL}$ 37\% $\mathrm{HCl}$ (SC Nordic Invest SRL, Cluj-Napoca) and $100 \mathrm{~mL}$ absolute EtOH (SC Nordic Invest SRL, Cluj-Napoca). The last two washings were repeated two times. In each washing the suspension was dispersed by magnetically stirring (5 min), sonicated (15 min) and centrifuged (5000 rpm for $15 \mathrm{~min}$ ) and the supernatant was eventually removed. In the third step, the resulted sludge was mechanically dispersed and sonicated (15 min) again in $200 \mathrm{~mL}$ of an EtOH/ $\mathrm{H}_{2} \mathrm{O}(1: 1$, vol.) solution. The resulting GO suspension was sealed and hold in a closed beaker (330 $\mathrm{ml}$ ), for 7 days in order to produce the isopycnic separation by sedimentation [45, 46]. Afterwards, using a syringe about $200 \mathrm{~mL}$ of this initial GO suspension was harvested from the upper part of the beaker suspension and dried in open atmosphere for 3 days. A dark-brown membrane-like GO solid was obtained. 


\subsection{Fabrication process of rGO flexible electrodes}

Flexible graphene oxide films (GOFs) supported on PET and glass substrates, as well as unsupported GO membranes (GOMs), were prepared from the synthesized GO powder. The steps for the fabrication process of rGO flexible electrodes from GOFs on PET are depicted in Fig. 1. Firstly, the GOFs were deposited on PET substrates (transparent and flexible sheets, $0.1 \mathrm{~mm}$ thick) by drop casting from the GO suspension (Figs. 1a, b, S1 Supporting Information). Two types of films with different thickness, single-deposited and double-deposited layered GOFs, were generated. The single-deposited GOFs were obtained spreading $0.06 \mathrm{~mL} / \mathrm{cm}^{2}$ of the GO suspension of $26 \mathrm{mg} / \mathrm{mL}\left(\mathrm{EtOH}: \mathrm{H}_{2} \mathrm{O}=1: 1\right.$, vol.) and drying during one day at room temperature (RT). The double-deposited GOFs were obtained spreading $0.06 \mathrm{~mL} / \mathrm{cm}^{2}$ of the GO suspension of $13 \mathrm{mg} / \mathrm{mL}\left(\mathrm{EtOH}: \mathrm{H}_{2} \mathrm{O}=\right.$ $1: 1$, vol.) after applying the monolayer leaving it one day at RT for drying. Once the GOFs were dried, the samples (GOFs/PET) were cut into $2.6 \times 7.6 \mathrm{~cm}^{2}$ rectangular pieces (Fig. 1c) and sandwiched between two microscope glass slides (1 mm thickness) by using two paper clips (Fig. 1d). Afterwards, the sandwich (glass/GOF/PET/glass) was exposed to a low-power visible laser beam (1W power, $450 \mathrm{~nm}$ wavelength) by using a MDL-F450-1000 laser system from CNI coupled to optic fibre and objective lens. The power loss in the optic fibre was about 30\%. The laser system was integrated in an engraving CNC machine for automatic irradiation of patterns (Fig. S2). The laser spot was modified in the 0.25 - $1.0 \mathrm{~mm}$ diameter range by focusing-defocusing of the beam with the aim to control the laser intensity reaching the sample (ca. $1.4-0.09 \mathrm{~kW} / \mathrm{cm}^{2}$, respectively). Laser reduced GOFs (LrGOFs) patterns were obtained by two different processing methods: (i) by irradiating adjacent locations (pixels) during a certain time (pixelated mode) (Fig. 1e), and (ii) by scanning the laser beam at constant speed (scanning mode) and using a reflective photomask (Fig. 1f). The photomask, located on the front glass slide, is used for avoiding thermal transient effects during beam acceleration at the beginning of the laser traces in scanning mode, being no longer necessary in pixelated mode. The laser exposure time per pixel and the separation between pixels used in pixelated mode ranged 1 - $500 \mathrm{~ms}$ and $0.1-0.2 \mathrm{~mm}$, respectively (Fig. 1e). On the other hand, the effect of laser scan speed was explored in the $10-1000 \mathrm{~mm} / \mathrm{min}$ range in scanning mode, keeping the distance between adjacent laser traces in $0.1 \mathrm{~mm}$ (Fig. 1f). Finally, the irradiated samples were immersed in water to remove the non-irradiated material (Fig. 1g) while the LrGOFs remained well adhered to the PET substrate since 
the interface between PET and GOFs was welded by the heat generated in GOFs. In this way, rGO flexible electrodes (LrGOFs/PET) were obtained (Fig. 1g).

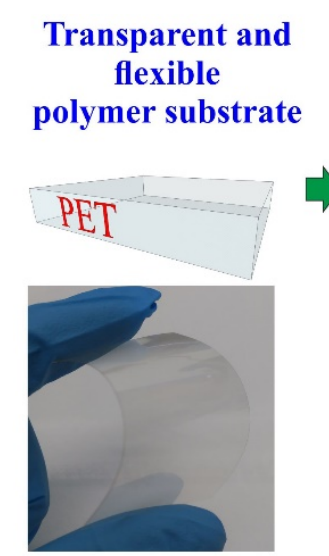

( a )

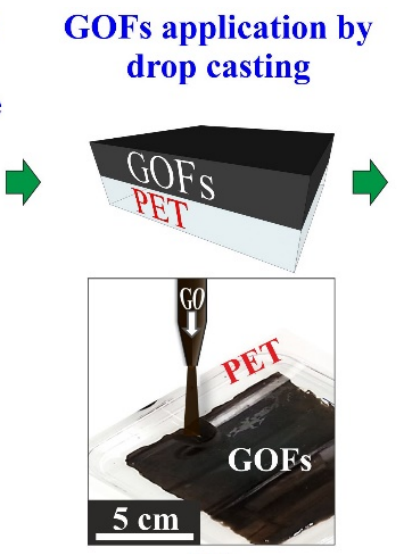

(b)

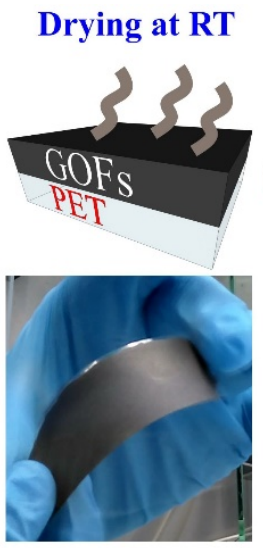

(c)
Sandwich between two glass wafers

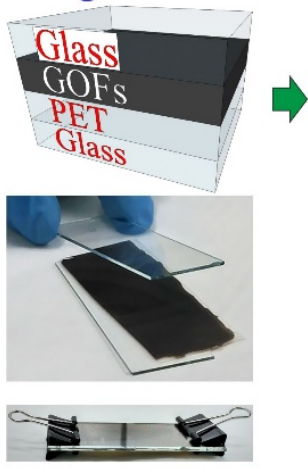

(d)

GOFs reduction by exposure to visible laser diode beam

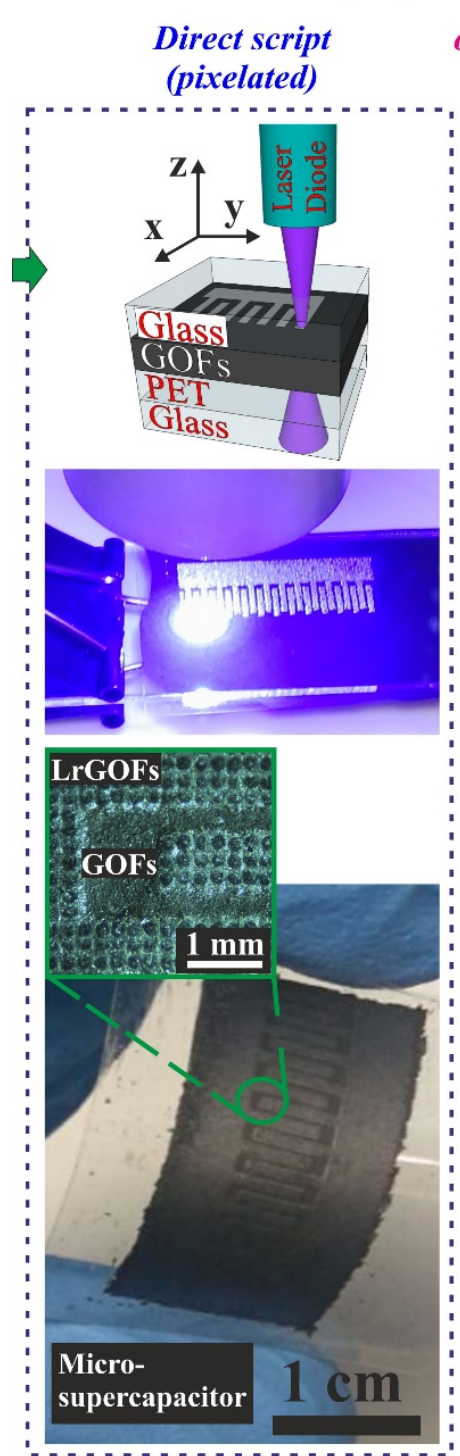

(e) or Scanning the laser beam and using photomask

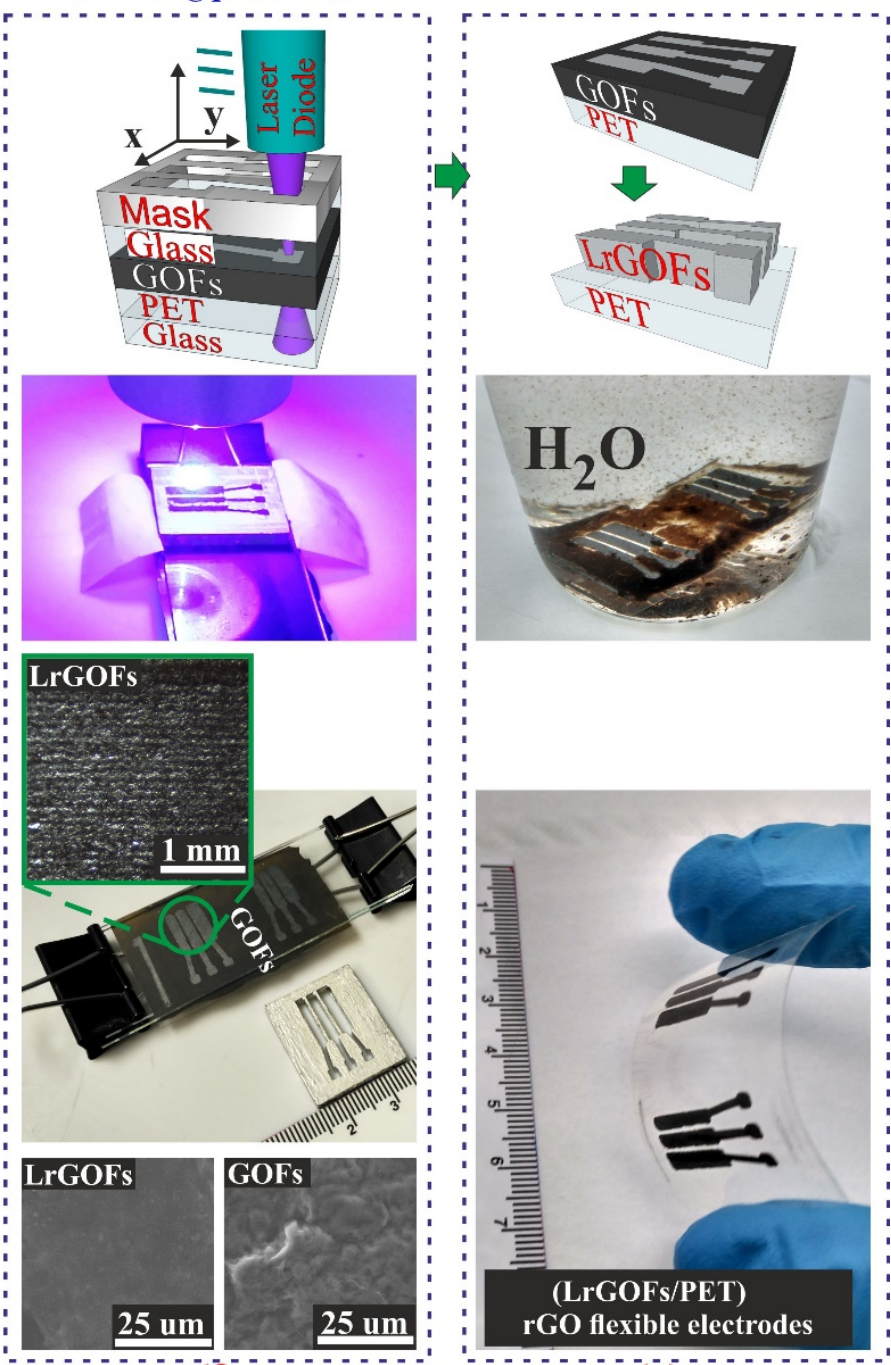

(f) non-reduced GOFs removing using water 
Fig. 1. Fabrication process of rGO flexible electrodes (LrGOFs/PET): (a) preparation of flexible PET substrate, (b) deposition of GOFs on PET by drop casting, (c) drying at RT, (d) sandwiching GOFs/PET between two glass slides, laser reduction by (e) direct laser writing of pixels or (f) scanning laser beam using a photomask, and (g) removing of nonirradiated GOF immersing the sample in water.

The same laser-reduction method was also implemented in GOFs/glass and GO membranes (Fig. 2). To prepare the GOFs/glass samples, single-deposited and doubledeposited GOFs were casted on microscope glass slides. The GOMs were obtained by peeling double-deposited GOFs from the glass support after drying. In this way, stable and freestanding GO films about $100 \mu \mathrm{m}$ in thickness were obtained and subjected to laser processing.

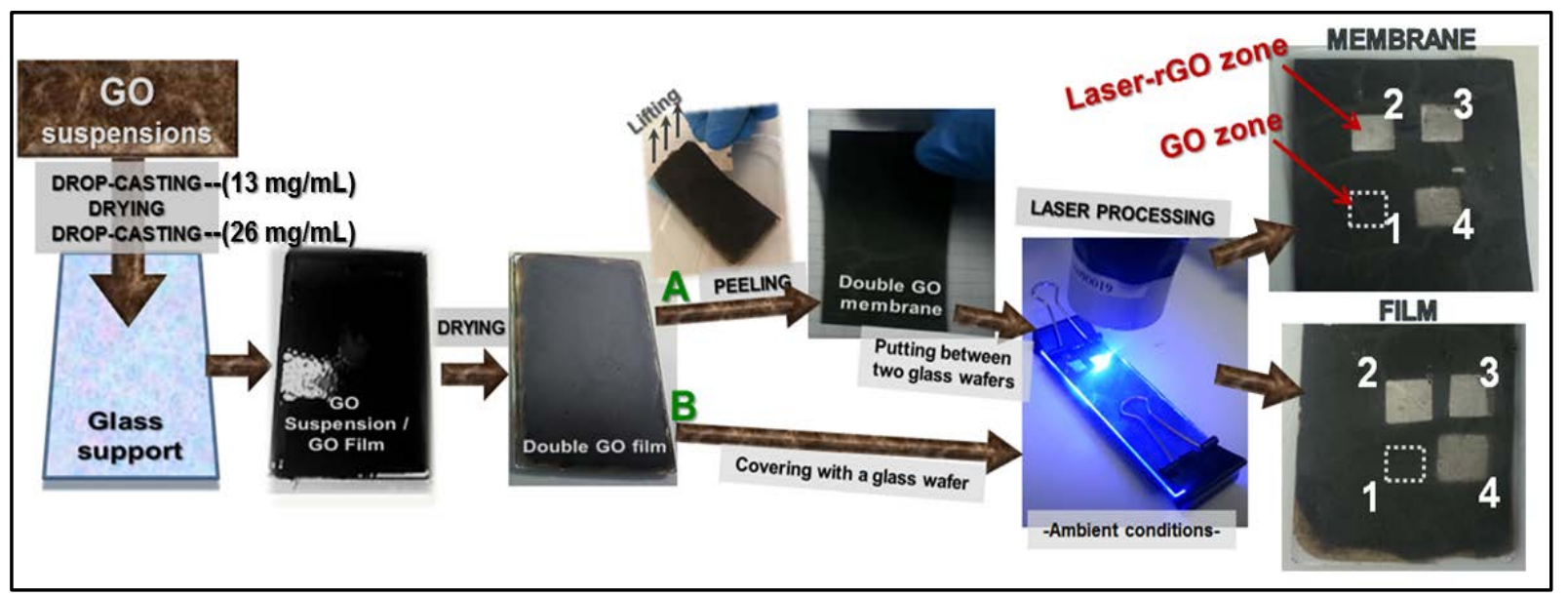

Fig. 2. Application of laser-reduction method in (A) GOMs and (B) double-depositedGOFs/glass. Numbered square zones are obtained by not-irradiation (zone 1) and scanning the laser beam with a $10 \mathrm{~mm} / \mathrm{min}$ speed, distance between laser traces of 0.1 $\mathrm{mm}$ and different laser spot sizes (0.25 mm (zone 2), $0.50 \mathrm{~mm}$ (zone 3) and $1 \mathrm{~mm}$ (zone 4)).

\subsection{Characterization techniques}

The morphology of the samples was studied by scanning electron microscopy (SEM) by using a Hitachi SU8230 CFE system working at $30 \mathrm{kV}$ accelerating voltage. Transmission electron microscopy (TEM) analyses were also performed by means of a Hitachi HD2700 CFE STEM microscope working at $200 \mathrm{kV}$ accelerating voltage. Scanning probe 
microscopy (SPM) analyses were performed through a Keysight 5100 system. Topographic maps were acquired by intermittent contact mode using silicon tips with less than $10 \mathrm{~nm}$ apex. The SPM data was processed with MountainsMap 7.4 software from Digital Surf. Raman spectra were recorded in backscattering geometry by using a multilaser confocal Renishaw InVia Reflex Raman spectrometer equipped with a Leica 100X (NA 0.75) microscope objective, a 1800 lines/mm grating, and an external laser with an emission wavelength of $532 \mathrm{~nm}$. During the spectra acquisition, a power of $150 \mathrm{~mW}$ incident on the sample was employed. The spectral resolution was about $4 \mathrm{~cm}^{-1}$. Nondestructive depth profiling was obtained by acquiring Raman spectra as the laser focus was automatically moved by a $1 \mu \mathrm{m}$ step deeper into each investigated sample, starting with the sample surface. Fourier transform infrared (FTIR) spectra were recorded in the range $4000-700 \mathrm{~cm}^{-1}$ with a spectral resolution of $4 \mathrm{~cm}^{-1}$ by using a Jasco IRT-5000 FTIR microscope coupled to Jasco FT-IR-6000 spectrometer in reflection configuration, using a $\times 32$ Cassegranian objective and imaging samples areas of $\sim 50 \times 50 \mu^{2}$. X-ray diffraction (XRD) analysis was carried out with a Shimadzu XRD-6000 diffractometer using $\mathrm{CuK} \alpha$ radiation $(\lambda=1.54 \mathrm{~nm})$, with Ni-filter. The diffractograms were recorded in the $2 \theta$ range from $5^{\circ}$ to $70^{\circ}$ with a speed of $2^{\circ} / \mathrm{min}$.

The electrical resistance (sheet resistance) of GOFs/PET, GOFs/glass and GOMs after the laser reduction (LrGOFs/PET, LrGOFs/glass and LrGOMs) was measured using micro-four-probe van der Pauw method on irradiated $5 \times 5 \mathrm{~mm}^{2}$ square areas. The corners of irradiated areas were coated with silver paste in order to obtain good electrical contacts for the electrical characterization. Simulation of the laser-reduction method was accomplished using COMSOL Multiphysics 5.4 software in order to simulate the temperature evolution in the GOFs when irradiated by laser. The 2D heat equation was solved in simple models composed of double-deposited GOFs (100 $\mu \mathrm{m}$ thick) casted on $100 \mu \mathrm{m}$ thick PET substrate, sandwiched between two $1 \mathrm{~mm}$ thick glass slides. The materials optical and thermophysical properties were obtained from the COMSOL materials library and ref. [27]. It should be also noted that optical losses in the glass slides were not taken into account in the simulations. Therefore, the simulated temperatures would be somehow overestimated though provide a helpful quasi-quantitative view of the laser-induced mechanisms.

The supercapacitive properties of the samples were analysed through cyclic voltammetry (CV) by means of a Keithley 2450-EC Electrochemistry Lab System. The samples were used as working electrode with a geometrical area of $0.5 \mathrm{~cm}^{2}$ in a three-electrode cell. 
The measurements were carried out with an $\mathrm{Ag} / \mathrm{AgCl}$ reference electrode (3M $\mathrm{NaCl}$ internal solution) and a Pt counter electrode in a $1 \mathrm{M} \mathrm{Na}_{2} \mathrm{SO}_{4}$ aqueous solution at room temperature. The mean areal capacitance of the flexible rGO electrodes (LrGOFs/PET) was calculated at different scan rates according to the following equation [47]:

$$
C_{\mathrm{A}}=\frac{\oint I(V) d V}{2 \Delta V v A}
$$

Where $C_{\mathrm{A}}$ is the areal capacitance, the integral in the numerator represents the area enclosed in the CV plot (anodic-cathodic charges), $\Delta V$ is the voltage window, $v$ is the voltage scan rate and $A$ is the area of the active material during the measurement. Additional galvanostatic charge-discharge (GCD) experiments were also performed to supercapacitor devices using the same voltage window. The areal capacitance was calculated through the equation:

$C_{A}=\frac{I t_{d}}{A \Delta V}$

Being I the applied current and $t_{d}$ the discharge time. The capacitance retention (ratio of $C_{\mathrm{A}}$ of each cycle related to the first one) and the coulombic efficiency (ratio of discharge and charge times at each cycle) was also carried out along 1000 cycles.

The performance of the patterned electrodes as electrochemical sensor-like devices was assessed by cyclic voltammetry using an Autolab potentiostat (EcoChemie, The Netherlands) controlled by Nova 2.1 software. A three-electrode electrochemical configuration was also used including working, counter and pseudo-reference electrodes made of rGO. Copper wires were glued to the patterned electrode pads using silver paint. A vinyl ring was stuck on top of the electrodes that allowed for placing and holding 10 $\mu \mathrm{l}$ of the measuring solution coating the electrode area. All measurements were carried out in $0.1 \mathrm{M} \mathrm{KCl}$ solutions containing $1 \mathrm{mM}$ ferrocene-methanol or $1 \mathrm{mM}$ ferricyanide redox probes.

\section{Results and discussion}

\subsection{Physical mechanism of laser-reduction method}

As explained above, the GO material synthesized for the preparation of GOF and GOM used in laser reduction experiments was obtained by a versatile sono-chemical oxidation method different from the well-known Hummers method. This procedure involved graphite as the main precursor, $\mathrm{H}_{2} \mathrm{SO}_{4}$ as reaction medium, $\mathrm{KMnO}_{4}$ as oxidative agent and $\mathrm{H}_{3} \mathrm{PO}_{4}$ as excessive-oxidation protective agent [46]. Besides, adjusted synthesis 
parameters as lower oxidation temperature and higher reaction time, efficient sonowashing processes and distinctive post-synthesis GO separation method performed in a homogeneous ethanol-water mixture were used [45]. In this way, a GO suspension with chemical properties prone to obtain stable GOF and GOM was obtained.

The main key of this laser-reduction method consists in sandwiching the sample (GOFs/PET, GOFs/glass, or GOMs) between glass slides while being irradiated by a lowpower visible laser beam (Fig. 3a) in order to generate a low oxygen-content atmosphere during the laser-induced GOFs reduction. Simulated temperature distributions show that the forward-facing glass cover allows the visible laser energy to be absorbed just by the GOF, generating a heat wave that propagates into the film and substrate (Fig. 3b, c). As observed, the maximum temperature is developed in the glass-GOF interface, reaching hundreds of $\mathrm{K}$ in the region adjacent to the GOF. The maximum temperature increases with the accumulated energy in the GOF, which is obtained by increasing the exposition time in pixelated mode and with the decrease of the scan speed and / or the laser spot diameter. This leads to the development of more than a thousand of $\mathrm{K}$ in the most extreme conditions (Fig. 3d). In the used range of experimental conditions, the reached maximum temperature in pixelated mode is about $650 \mathrm{~K}$, whereas it raises up to ca. $1450 \mathrm{~K}$ in scanning mode at $10 \mathrm{~mm} / \mathrm{min}$, below the glass melting point (ca. $1700-1900 \mathrm{~K}$ ). It is worth noting that, in scanning mode, the maximum temperature developed (peaks at ca. $20 \mathrm{~s}$ in Fig. 3d) are somewhat overestimated due to finite dimension of the modelled system (also observed by the increasing temperature in time after the temperature peak). The high temperatures developed in the GOF leads to its thermal reduction, as it will be demonstrated in Section 3.2. Moreover, the melting temperature of PET (ca. $530 \mathrm{~K}$ ) can be overpassed, provoking its partial melting in the GOF-PET interfase. After cooling and resolidification, PET and laser reduced GOF (LrGO) become joined providing adherence and mechanical stability to the LrGO. Despite the development of such high temperatures in the PET substrate, the LrGO-PET system maintained the mechanical integrity in the explored range of experimental parameters. Besides, it is worth to note that similar numerical results are obtained in double-deposited and single-deposited GOFs. 


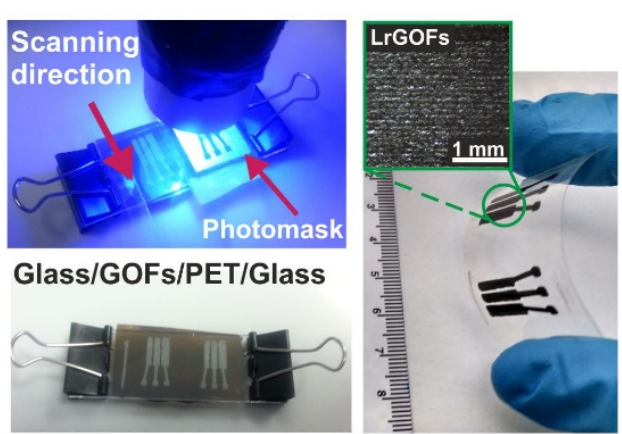

(a)

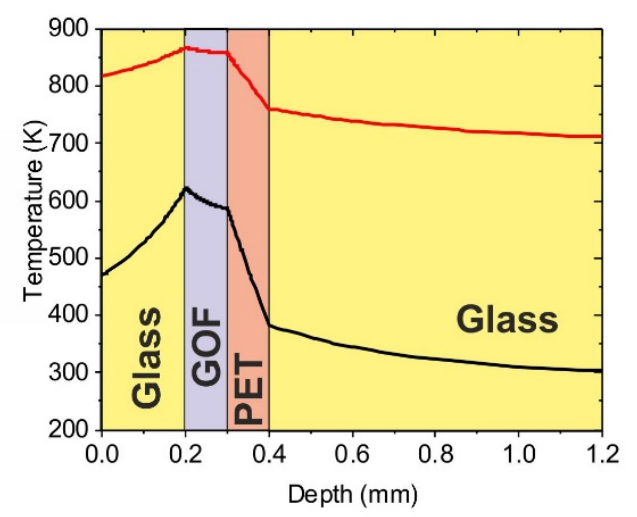

(c)

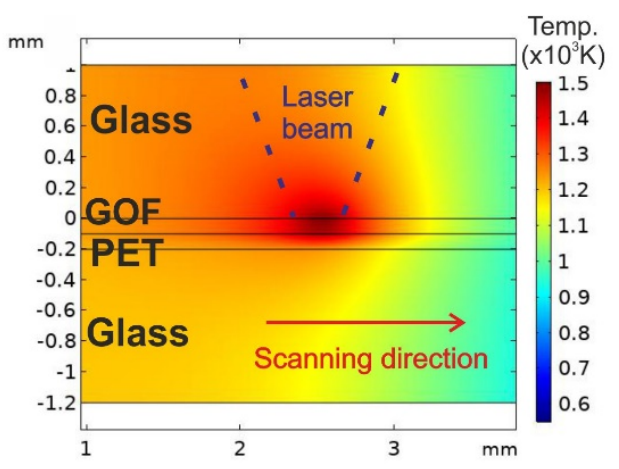

(b)

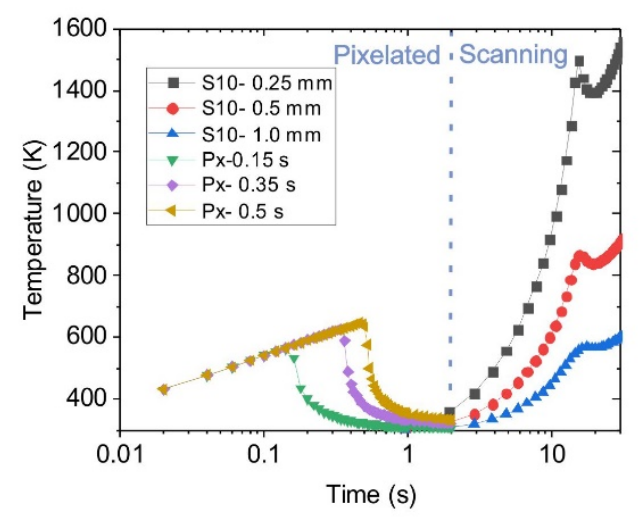

(d)

Figure 3. (a) Image of the fabrication of rGO flexible electrodes obtained by scanning mode (through a photomask) on a glass/double-deposited GOFs/PET/glass sandwich. (b) Simulation of the temperature distribution (cross-section view) by laser exposure of the system developed in (a). The thickness of double-deposited GOF is $100 \mu \mathrm{m}$, the laser spot diameter and scan velocity are $0.25 \mathrm{~mm}$ and $10 \mathrm{~mm} / \mathrm{min}$, respectively. (c) Simulation of the temperature profile in depth (at the maximum temperature instant) when the sandwich of (a) is irradiated with a laser spot of $0.5 \mathrm{~mm}$ in scanning mode with $10 \mathrm{~mm} / \mathrm{min}$ speed (red) and in pixelated mode with 0.35 s exposure time (black). (d) Simulation of time evolution of temperature at one location on the glass-GOF interface when the sandwich of (a) is irradiated in scanning mode with $10 \mathrm{~mm} / \mathrm{min}$ speed at different spot diameters and in pixelated mode with $0.5 \mathrm{~mm}$ spot at different exposure times.

The effects of laser irradiation on GOFs were significantly different depending on the presence or not of the glass cover. The direct in-air action (i.e. without covering with the glass slide) was carried out irradiating a $10 \times 1 \mathrm{~mm}^{2}$ rectangular area of single-depositedGOF/glass with $0.25 \mathrm{~mm}$ spot, distance between beam traces of $0.1 \mathrm{~mm}$, and a beam speed of $250 \mathrm{~mm} / \mathrm{min}$ (zone 1 from Figs. 4a-d). As can be observed, remarkable vaporization provoked a rectangular channel in which transparent glass support is 
revealed surrounded with redeposited material (Fig. 4b) due to high mass loss of the irradiated GO. According to the literature, volatile compounds as $\mathrm{H}_{2} \mathrm{O}, \mathrm{CO}_{2}, \mathrm{CO}$ or/and even $\mathrm{SO}_{2}$ and other small gas molecules are created during a thermal treatment due to the decomposition/burning of carbon mesh as well as oxygen-containing functional groups of GO [48-50]. This oxidative process is directly driven by the presence of ambient oxygen and the increase of material temperature (exothermal process). Taking into account these premises, the process of gas generation (i.e. mass lost) that take place in open-air laser irradiation of a GOF could be represented as in Figure 4d. Generated gas molecules, which are outlined for simplicity only on the right side of the formed carbon wall (Fig. 4d - left side), get out in a massive amount by a large front-action due to the accentuated burning process causing the displacement of debris material to the trace boundaries. 3D optical microscopic images of Fig. 4d (right side) show the generated carbon wall that gets out from the plane of irradiated rectangular zone.

A completely different behavior occurs when the same laser treatment was performed through a second glass slide tightly placed on GOF-glass (zones 2-4 of Fig. 4a and Fig. S3). In this case, there is a tiny space between the GO layer and the upper-covering glass slide. By this approach, two processes that are more favored to take place during GO reduction instead of oxidation could be identified: (i) a less amount of oxygen is present near to the irradiated GOF region; (ii) the generated gases (i.e. $\mathrm{H}_{2} \mathrm{O}, \mathrm{CO}_{2}, \mathrm{CO}, \mathrm{SO}_{2}$ ) formed during the irradiation process produce an overpressure that blocks the input of molecular oxygen from the environment (air). A brief sketch of the described steps is illustrated in Fig. 4e - left side. In this way, an in situ local inert atmosphere isolated from the surrounding oxidative one is obtained. As it will be described in Section 3.3, the irradiated regions (zones 2-4 of Fig. 4a and Fig. S3) reveal orders of magnitude lower electrical resistance than the non-irradiated GOF (Table 1). This fact points to laserinduced reduction mechanisms of glass-covered GO material. In this case, the irradiated GO does not stand out from the initial GO material plane (Figure 4e - right side). 


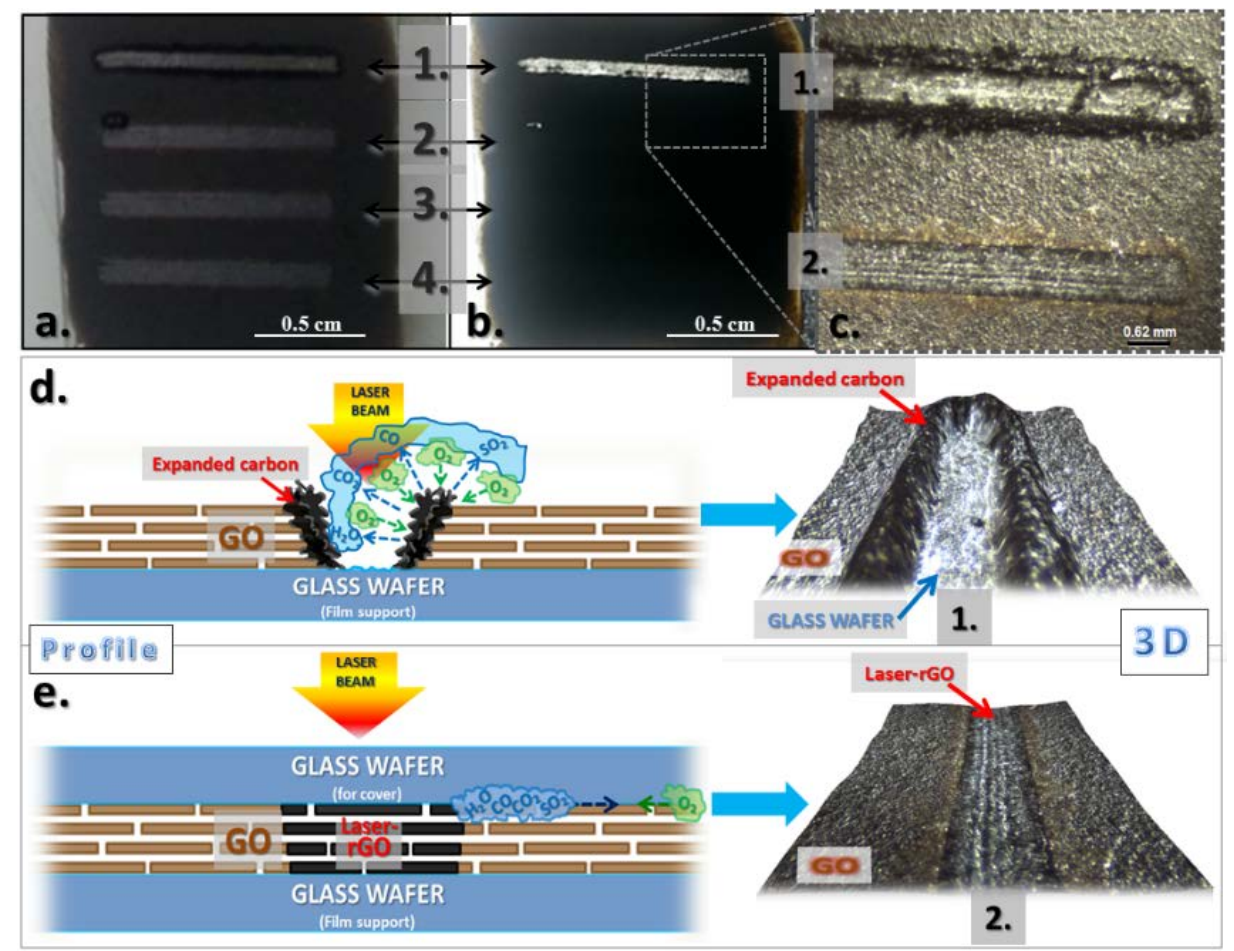

Figure 4. (a) Front and (b) rear illuminated pictures of single-deposited-GOF/glass sample on which $1 \times 10 \mathrm{~mm}^{2}$ rectangular areas were processed by laser scanning mode. Zone 1 was obtained in uncovered GOF (without the forward-facing glass slide) and zones 2-4 on covered ones. The zones 1-4 were obtained by scanning a laser beam with $0.25 \mathrm{~mm}$ diameter, using $0.1 \mathrm{~mm}$ distance between consecutive traces, and different scan speeds (250 (zones 1 and 2), 500 (zone 3) and 1000 $\mathrm{mm} / \mathrm{min}$ (zone 4)). (c) Optical microscopy image of zones 1 and 2. (d, e) Sketch of proposed mechanisms during GO laser irradiation (left) and 3D optical microscopic images (right) of irradiated zones (d) without glass covering and (e) covered ones.

\subsection{Sctructural analysis of GOFs and LrGOFs}

Scanning probe microscopy was used to visualize the GO sheets of the suspension obtained by sono-chemical process (Fig. 5a). The presence of GO sheets with a thickness of 1.0-1.2 nm confirms the efficiency of oxidative exfoliation of graphite leading to GO monolayers. SPM and TEM images revealed GO sheets with planar size of about tens of $\mu \mathrm{m}^{2}$ (Figs. 5a, b). No remarkable structural defects were detected in the GO plates besides furrows and irregular edges. Surface and cross-section regions of non-irradiated and irradiated GO membranes were also investigated by SEM (Figs. 5d, e and Table S1). A structure composed of stacked GO sheets can be inferred from surface of GOM (Figs. 5ce). As observed, the spatial arrangement of the GO sheets gives rise to the formation of 3D layered structures. This layered structure is more evident by analysing the cross- 
section, in which overlapped planar features are visible leading to the formation of a compact film (Table S1). A decrease of thickness from ca. $100 \mu \mathrm{m}$ of non-irradiated GOM to 66-80 $\mu \mathrm{m}$ of irradiated GOMs was evidenced (Table S1), pointing to an increase of compactness of the material. EDX analyses performed on GOMs allow the investigation of the atomic C/O ratio (Table S1). Non-irradiated GOM presents a C/O ratio of ca. 1.5. The highest value of about 4.0 was obtained in the sample irradiated with $0.25 \mathrm{~mm}$ spot and $10 \mathrm{~mm} / \mathrm{min}$ laser scanning speed. Maintaining the rest of parameters, values of about 2.5 and 2.3 were obtained by using larger spots, 0.5 and $1.0 \mathrm{~mm}$, respectively. These results prove (i) the loss of oxygen (reduction) caused by laser irradiation, and (ii) a higher degree of reduction with the increase of laser intensity (decrease of spot dimensions), due to the development of larger temperatures. Analogous results are obtained with GOFs (not shown).
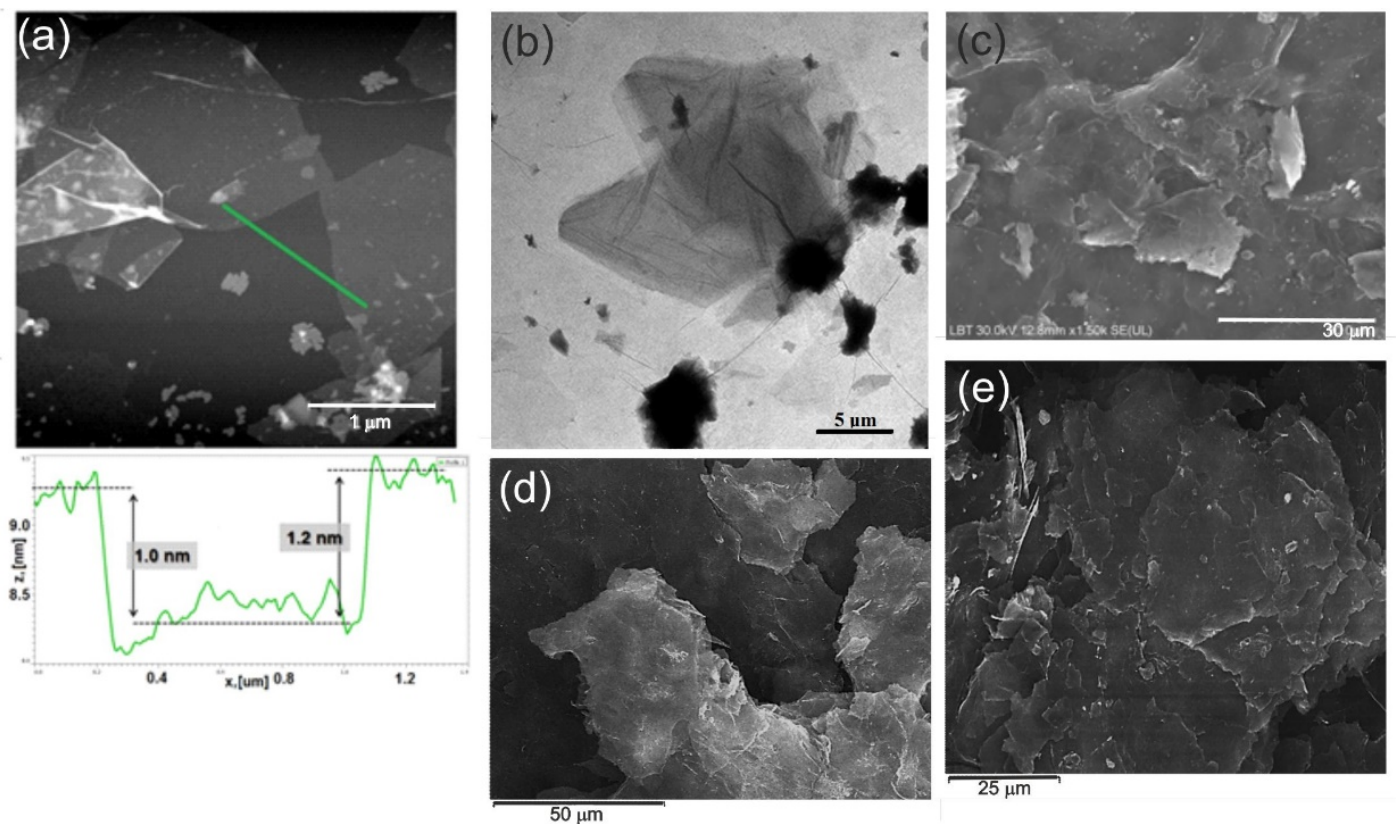

Figure 5. (a) SPM and (b) TEM cross-section images of initial GO material. A topographic profile that corresponds to the marked line from the upper image is also shown in (a). SEM images of GOM surface (c) non-irradiated, and irradiated with 10 $\mathrm{mm} / \mathrm{min}$ scan speed and (d) $0.5 \mathrm{~mm}$ and (e) $0.25 \mathrm{~mm}$ spot size.

The chemical composition of the obtained materials was studied by Fourier transform infrared spectroscopy. FTIR spectra of GOM and LrGOM are presented in Fig. 6. The spectrum of GO is dominated by several spectral features as follows: the absorption at 
$3400 \mathrm{~cm}^{-1}$ attributed to the stretching mode of $-\mathrm{OH}$ group in $\mathrm{H}_{2} \mathrm{O}$, the band given by the $\delta\left(\mathrm{H}_{2} \mathrm{O}\right)$ mode at $1635 \mathrm{~cm}^{-1}$, the band at $1760 \mathrm{~cm}^{-1}$ attributed to $\mathrm{C}=\mathrm{O}$ stretching vibrations from carbonyl and carboxyl groups, and the absorption signals at 1320, 1225 and 1070 assigned to $\mathrm{C}-\mathrm{OH}$ bending, $\delta(\mathrm{C}=\mathrm{C})$ mode and $\mathrm{C}-\mathrm{O}$ functional groups, respectively [51]. As can be observed from Fig. 6a, the intensity of the absorption bands drastically decreased after laser irradiation. Moreover, the bands at 1320 and $3400 \mathrm{~cm}^{-1}$ disappeared indicating the removal of C-OH functional groups and intercalated $\mathrm{H}_{2} \mathrm{O}$ molecules. This behaviour was also proved by the IR spectral maps recorded at the boundary between deposited GOM and LrGOM region (Fig. 6b). As observed, the intensity of C-OH and $\mathrm{H}_{2} \mathrm{O}$ bands is significantly lower in the irradiated region than in the non-irradiated one. On the other hand, the intensity of $\mathrm{C}=\mathrm{O}$ stretching band $\left(1760 \mathrm{~cm}^{-1}\right)$ was quite stable after irradiation, only showing a narrowing of the band, the most probably due to the shrinkage of $\mathrm{C}=\mathrm{O}$ bonds distribution (Fig. 6a). 


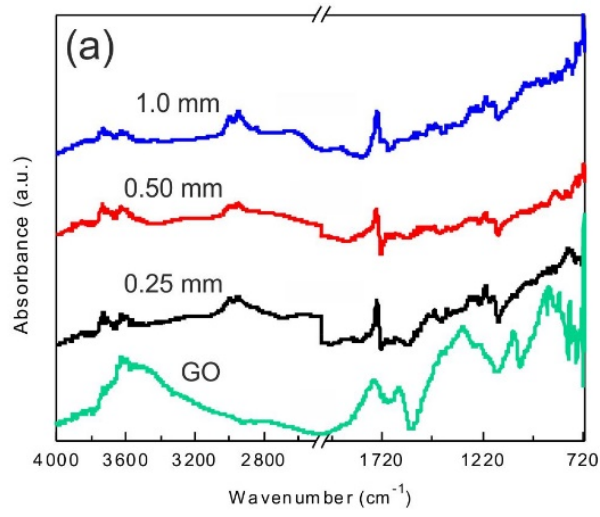

(b)
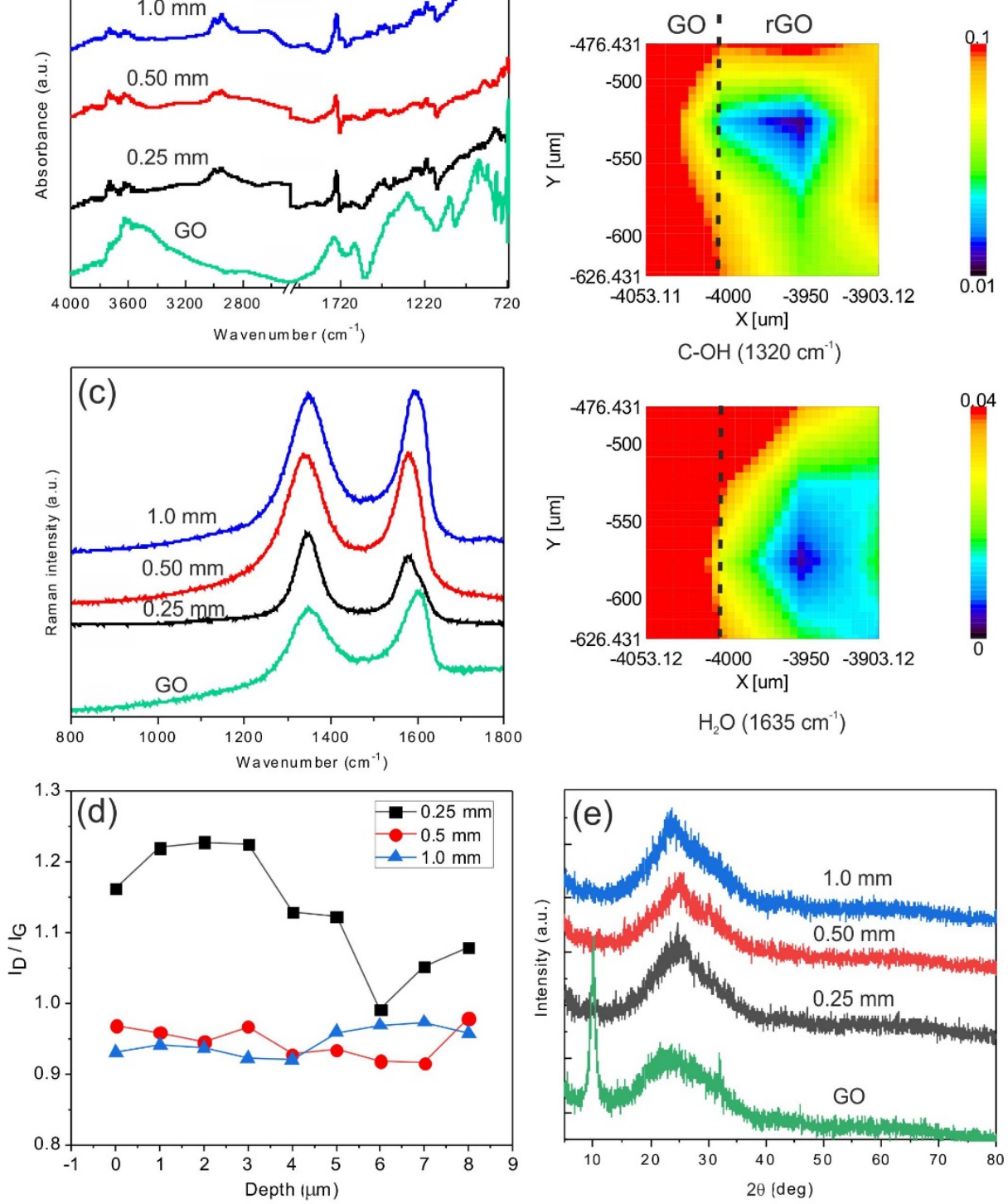

Figure 6. (a) FTIR spectra of the membrane, not-irradiated (GO) and irradiated with 0.25 , 0.5 and $1 \mathrm{~mm}$ laser spots, $10 \mathrm{~mm} / \mathrm{min}$. (b) Characteristic FTIR bands intensity distribution of two representative bands, C-OH $\left(1320 \mathrm{~cm}^{-1}\right)$ and $\mathrm{H}_{2} \mathrm{O}\left(1635 \mathrm{~cm}^{-1}\right)$, recorded at the boundary between the non-irradiated GOM and LrGOM (left side and right side of the dashed line, respectively) with $0.25 \mathrm{~mm}$ laser spot. Note that the images were recorded in the same area. (c) Raman spectra of the treated membranes with different laser spot size. (d) Depth profiling representation of the $\mathrm{I}_{\mathrm{D}} / \mathrm{I}_{\mathrm{G}}$ ratio for the three laser treatments presented in (c). Note that the depth profiles are the average value of four independent measurements. (e) X-ray diffraction pattern obtained from the irradiated membrane with the different laser spots. 
Raman spectra of all GOFs / GOMs and LrGOFs / LrGOMs samples were recorded and selected spectra are illustrated in Fig. 6c. Two well-known features appear in all spectra: the $\mathrm{D}$ band, around $1350 \mathrm{~cm}^{-1}$, which arises due to structural disorder in graphitic structure, and the $\mathrm{G}$ band, at ca. $1590 \mathrm{~cm}^{-1}$, which corresponds to the first order scattering of $E_{2 g}$ phonons from $\mathrm{sp}^{2}$ carbon bonds contained in the material [52-54]. Deconvolution of the spectra exhibits additional bands (I located at 1100-1200 $\mathrm{cm}^{-1}$, D'” at 1500-1550 $\mathrm{cm}^{-1}$, and D' at 1600-1615 $\mathrm{cm}^{-1}$ ) as witnessed in Fig. S4a [55]. The observed broadening of G-D' signal up to $1650 \mathrm{~cm}^{-1}$ (Fig. 6c) would point to the presence of nanocrystalline graphitic domains, that is, to high density of defects in GO structure. In addition, the G peak appears slightly shifted to lower wavenumber values after laser reduction processes, from $1588 \mathrm{~cm}^{-1}$ (GO) towards $1580 \mathrm{~cm}^{-1}$ (for $0.25 \mathrm{~mm}$ ). This shift would indicate a decrease of defects density [53], probably by graphene domains restoration, with the increase of the accumulated energy of the laser treatment. It is worth noticing that the chemical path during GO deoxidation, leading to either the formation of structural defects or graphene domains restoration, strongly depends on its particular composition [34]. On the other hand, it is well-known that the intensity ratio between the D and G bands can provide information regarding defects in graphene material. Previous results point to a relation between the GO reduction and the increase of the $I_{D} / I_{G}$ intensity ratio [56]. As can be seen from the calculated $\mathrm{I}_{\mathrm{D}} / \mathrm{I}_{\mathrm{G}}$ ratios (Fig. $6 \mathrm{~d}$ ), the highest value was obtained in the GOM reduced with a $0.25 \mathrm{~mm}$ spot size, followed by those obtained with $0.5 \mathrm{~mm}$ and $1.0 \mathrm{~mm}$ spots which show very similar values. Given that the initial material presents nanocrystalline graphitic domains and high density of defects, the increase of $I_{D} / I_{G}$ intensity ratio would also indicate a decrease of defects density by graphene restoration [53]. This effect was also verified by calculating the ratio of the integrated areas of $\mathrm{D}$ and G bands (D/G) (Fig. S4b). Moreover, the study of 2D band can further confirm our assumptions since it is also sensitive to the defects of graphene-based materials [57]. Fig. S4c displays the higher wavenumber region of the Raman spectra showing the $2 \mathrm{D}$ and $D+G$ peaks. By comparative consideration of the 2D band intensities, it can be observed that the sample LrGOM irradiated with $0.25 \mathrm{~mm}$ spot size exhibits the highest intensity demonstrating again the existence of less defects in the rGO structure. In the case of the samples irradiated with 0.5 and $1 \mathrm{~mm}$ spot size, the spectral nature of the 2D band is revealed to be very similar between them and with that of the GO. Additionally, the D/D' ratio of integrated areas was also calculated for attaining additional knowledge about the 
nature of the defects present in the LrGO samples. As witnessed in Fig. S4b, the D/D' ratio shows a steady decrease from 0.16 (GOM) to 0.05 (LrGOM irradiated with 0.25 $\mathrm{mm}$ ) pointing to a transition from vacancy predominated structure to a $\mathrm{sp}^{3}$ defect dominated one with increasing laser accumulated energy [55]. Noteworthy, depth profiling analysis reveals some decrease of the $\mathrm{I}_{\mathrm{D}} / \mathrm{I}_{\mathrm{G}}$ ratio in depth as observed in LrGOM irradiated with $0.25 \mathrm{~mm}$ spot size after 3-4 $\mu \mathrm{m}$ in depth, whereas the ratio is pretty unchanged within 6-8 $\mu \mathrm{m}$ in depth in membranes irradiated with 0.5 and $1.0 \mathrm{~mm}$ spot. XRD data of representative GOM samples are shown in Fig. 6e. The diffractograms reveal the well-known characteristic peak of GO [46] that usually appears at ca. $2 \theta=10^{\circ}$, which disappears after laser irradiation. Besides, a broad band can be seen at about $2 \theta=$ $25^{\circ}$ which is associated with the formation of graphite after opening of the epoxy rings and removal of other oxygen moieties in GO structure [52]. Therefore, and in concordance with FTIR and Raman spectroscopy results, a laser-induced reduction of the GOMs is obtained. By comparing the X-ray diffractograms, no evident differences were observed in LrGO samples.

\subsection{Electrical and electrochemical characterization}

The electrical response of reduced graphene oxide materials is directly linked to its reduction degree and the density of defects, since it depends on graphene domain configuration and electron scattering processes $[58,59]$. To study the electrical response of LrGOFs as a function of laser speed in scanning mode, $10 \times 1 \mathrm{~mm}^{2}$ rectangular areas of single-deposited-GOFs/glass were irradiated by scanning the laser beam $(0.25 \mathrm{~mm}$ spot diameter), with different laser scan speeds (250, 500 and $1000 \mathrm{~mm} / \mathrm{min}$ ) at $0.1 \mathrm{~mm}$ distance between laser traces (Figs. 4, S3). As previously detailed, an additional rectangular area was irradiated rastering the laser beam at $250 \mathrm{~mm} / \mathrm{min}$ but without being sandwiched between two glass slides (zone 1 of Fig. 4). As observed in Table 1, the GOFs irradiated without glass cover presents very high resistance due to GO partial oxidation and decomposition. To the contrary, the zones irradiated between the glass slides reveal a decrease of the electrical resistance due to partial reduction of the GO material without significant degradation. As observed, the lower the scan speed (i. e. higher accumulation of laser energy), the lower is the measured resistance (i. e. larger GO reduction degree). 


\begin{tabular}{|c|c|c|c|}
\hline \multirow{2}{*}{$\begin{array}{l}\text { Sample } \\
\text { LrGOFs/glass } \\
10 \times 1 \mathrm{~mm}^{2} \\
(\text { Fig. } 4 \mathrm{a})\end{array}$} & \multicolumn{2}{|c|}{ Laser parameters } & \multirow{2}{*}{$\begin{array}{l}\text { Electrical resistance } \\
\mathbf{R}(\mathbf{\Omega}) \\
\text { Electrical resistance between } \\
\text { extremes of irradiated areas. }\end{array}$} \\
\hline & $\begin{array}{l}\text { Spot } \\
\text { (mm) }\end{array}$ & $\begin{array}{l}\text { Scan speed } \\
\text { (mm/min) }\end{array}$ & \\
\hline $\begin{array}{l}1 \text { (without glass } \\
\text { cover) }\end{array}$ & 0.25 & 250 & $>10^{6}$ \\
\hline 2 & \multirow{3}{*}{0.25} & 250 & 535 \\
\hline 3 & & 500 & 660 \\
\hline 4 & & 1000 & 1162 \\
\hline
\end{tabular}

Table 1. Electrical resistance evolution as function of the laser scan speed with spot of $0.25 \mathrm{~mm}$ in diameter for LrGOFs/glass. The distance between beam traces was $0.1 \mathrm{~mm}$. The resistance of the initial material is larger than $1 \mathrm{M} \Omega$.

Additional studies were also carried out in order to assess the influence of the spot size on the electrical response of GOFs/glass and LrGOMs. In this case, $5 \times 5 \mathrm{~mm}^{2}$ square areas were irradiated scanning the laser beam at $10 \mathrm{~mm} / \mathrm{min}$ speed, $0.1 \mathrm{~mm}$ distance between beam traces and different beam spots (0.25, 0.5 and $1 \mathrm{~mm}$ ) (Table 2, Fig. 2). As observed, the increase of laser intensity by means of smaller spot dimensions at this low scan speed leads to a substantial decrease of the sheet resistance from more than $1 \mathrm{M} \Omega / \mathrm{sq}$ of initial material to just 8-9 $\Omega$ /sq in samples irradiated with $0.25 \mathrm{~mm}$ laser spot, corresponding to resistivity values of ca. $6 \times 10^{-4} \Omega \mathrm{m}$. To the best of our knowledge, these sheet resistance values are between the lowest ones obtained through laser processing of GO material as compared to literature [60-62]. The results of the electrical measurements (electrical resistance and sheet resistance) correlate with those of the Raman analysis, according to that the most intense graphene restoration process occurs for the GOM reduced with the smallest spot diameter favouring thus the electrical conduction of the material. 


\begin{tabular}{|c|c|c|c|c|}
\hline \multirow{2}{*}{$\begin{array}{l}\text { Samples } \\
5 \times 5 \mathrm{~mm}^{2} \\
\text { (Fig. 2) }\end{array}$} & \multicolumn{2}{|c|}{ Laser parameters } & \multicolumn{2}{|c|}{$\begin{array}{l}\text { Sheet resistance, Rs }(\Omega / s q) \\
\text { (Van der Pauw method) }\end{array}$} \\
\hline & $\begin{array}{l}\text { Spot } \\
\text { (mm) }\end{array}$ & $\begin{array}{l}\text { Scan speed } \\
(\mathrm{mm} / \mathrm{min})\end{array}$ & $\begin{array}{l}\text { LrGOMs } \\
\text { (double-deposited) }\end{array}$ & $\begin{array}{l}\text { LrGOFs/glass } \\
\text { (double- deposited ) }\end{array}$ \\
\hline 1 & \multicolumn{2}{|c|}{ No irradiation } & $>10^{6}$ & $>10^{6}$ \\
\hline 2 & 0.25 & \multirow{3}{*}{10} & 8 & 9 \\
\hline 3 & 0.50 & & 32 & 23 \\
\hline 4 & 1.0 & & 320 & 135 \\
\hline
\end{tabular}

Table 2. Sheet resistance evolution as function of the laser spot size at $10 \mathrm{~mm} / \mathrm{min}$ scan speed for LrGOMs and double-deposited LrGOFs/glass. The distance between laser traces was $0.1 \mathrm{~mm}$.

Finally, $5 \times 5 \mathrm{~mm}^{2}$ squared areas of GOFs/PET (both single-deposited and doubledeposited) sandwiched between the two glass slides were also irradiated in pixelated mode with $0.1 \mathrm{~mm}$ separation between pixels, and several exposure times (from $1 \mathrm{~ms}$ to $1 \mathrm{~s}$ ) in order to study the electrical response of LrGOFs/PET as a function of laser exposure time (Fig. 7). As shown, the sheet resistance decreases with laser exposure time per pixel reaching a minimum at $0.2 \mathrm{~s}$ and $0.5 \mathrm{~s}$ for single-deposited and double-deposited GOFs, respectively. The lowest sheet resistance value, achieved in double-deposited LrGOFs, is $7 \Omega$ /sq. Higher exposure times provoke the crack formation in the irradiated material due to large thermal gradients and the onset of local melting-vaporization processes leading to an increase of the sheet resistance. 


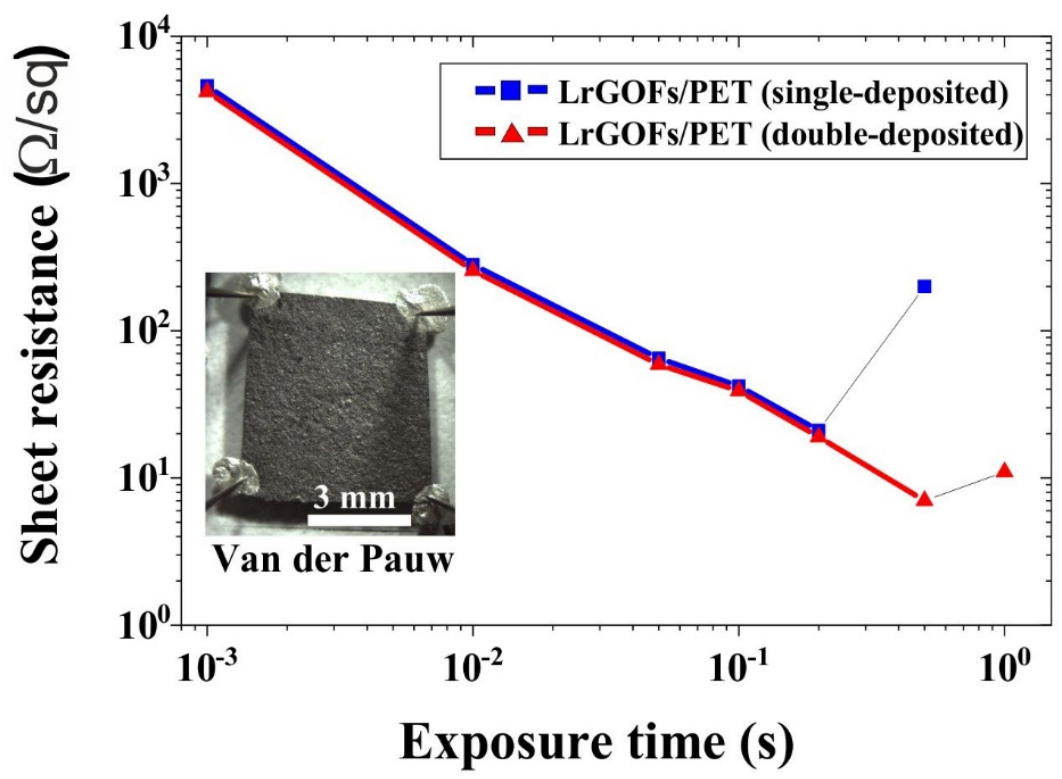

Figure 7. Evolution of LrGOFs sheet resistance as a function of time exposure in pixelated mode. The laser exposure conditiones for both single-deposited and double-depositedGOFs were: $0.25 \mathrm{~mm}$ spot diameter and $0.1 \mathrm{~mm}$ of separation between irradiated zones.

Electrochemical measurements of the electrodes with the lowest sheet resistance were carried out for testing their performance in supercapacitor and sensing applications. Figs. 8a, b display typical cyclic voltammetry plots of single-deposited and double-deposited LrGOFs/PET in $\mathrm{Na}_{2} \mathrm{SO}_{4}$ electrolyte showing the characteristic rectangular-like shape of capacitive materials. The pronounced peak appearing at about $+0.8 \mathrm{~V}$ is caused by the oxygen evolution due to water hydrolysis at the electrode/electrolyte interface. Besides, the observed tilting of the signal towards higher currents in the double-deposited LrGOFs indicates some leakage of charge probably due to crystalline defects and/or chemical reactions between the electrode and the electrolyte ions. This effect, not observed in single-deposited films, could point to slightly lower reduction degree in double-deposited GOFs. As observed in Fig. 8c, the areal capacitance of the electrodes decreases with the scan rate, showing the double-deposited electrode higher capacitance than the singledeposited one (ca. 27 and $10 \mathrm{mF} / \mathrm{cm}^{2}$ at $10 \mathrm{mV} / \mathrm{s}$, respectively). The obtained areal capacitance is comparable to that of similar systems reported in the literature [63, 64]. Fig. S5 shows cyclic voltammetry and galvanostatic charge-discharge performance of an interdigital microsupercapacitor fabricated in single-deposited-GOF/PET through pixelated mode. The stretched rectangular-like shape of voltammograms, without well- 
defined peaks, and the quasi-triangular nature of charge-discharge performance, without plateaus, point to non-faradaic processes as the origin of energy storage (Figs. S5a,b). The areal capacitance of the device is ca. $5.2 \mathrm{mF} / \mathrm{cm}^{2}$ at $10 \mathrm{mV} / \mathrm{s}(\mathrm{CV}$, Fig. S5b) and ca. $3.7 \mathrm{mF} / \mathrm{cm}^{2}$ at $0.2 \mathrm{~mA} / \mathrm{cm}^{2}$ current density (GCD, Fig. S5d), acting the non-irradiated GOF in gaps as porous separator (impregnated in $1 \mathrm{M} \mathrm{Na}_{2} \mathrm{SO}_{4}$ aqueous electrolyte). As expected, the areal capacitance decreases with increasing the potential scan rate (CV) and the applied current density (GCD). Fig. 8d shows typical galvanostatic charge-discharge cycling performance of the device. As observed, the device is flexible and its capacitance remains practically constant at the maximum value after 1000 cycles. On the other hand, the coulombic efficiency is maintained at ca. $80 \%$ in all the range, accounting for stable leakage processes and no significant formation of structural defects that influence electrochemical performance during cycling. The performance of the obtained device is comparable to the reported ones in the literature either by laser or other methods, being the range of reported capacitances pretty wide [63,65-66]. It must be noted that the performance of the devices not only depends strongly on the structural configuration of the material (specific surface area, nature of the porosity, presence of electroactive species, etc) but also on its thickness $[68,69]$.

Fig. 8e shows the electrochemical cell set-up used for carrying out the assessment of the rGO electrodes for sensing. Ferricyanide and ferrocene-methanol representative redox probes were tested whose corresponding electrochemical processes follow inner-sphere and outer-sphere redox mechanisms, respectively [70]. The former accounts for those redox processes that are sensitive to the surface composition of the electrode and could provide some information about oxygen-containing defects of the graphene material in contact with the measuring solution. From the results shown in Fig. 8f, both ferricyanide and ferrocene-methanol cyclic voltammetric process show a quasi-reversible behaviour with the peak potential separation between the anodic and cathodic signal being $193 \mathrm{mV}$ and $110 \mathrm{mV}$ (at $50 \mathrm{mV} / \mathrm{s}$ ), respectively. The estimated half-wave potentials for both species were $160 \mathrm{mV}$ and $28.5 \mathrm{mV}$ in the $0.1 \mathrm{M} \mathrm{KCl}$ background electrolyte. Both measurements are in accordance with a conductive carbon-like electrode surface with a low density of oxygen-containing groups [70,71]. 


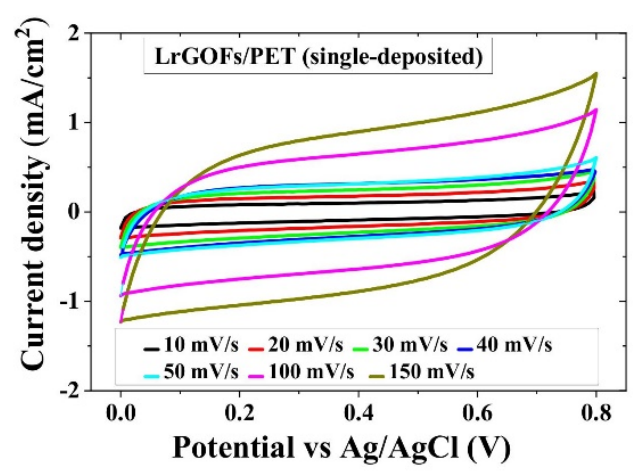

(a)

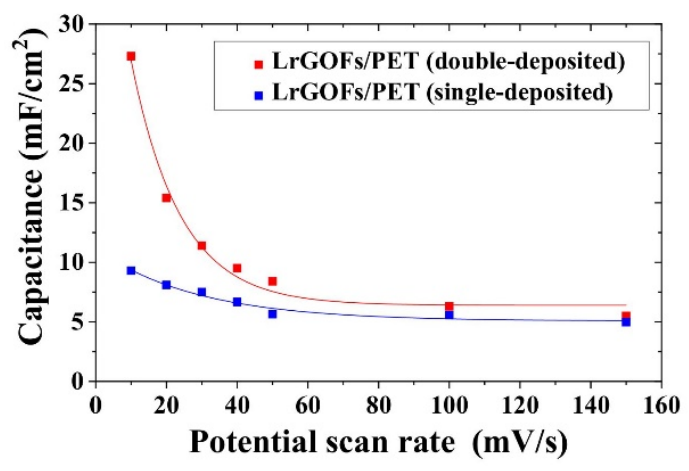

(c)

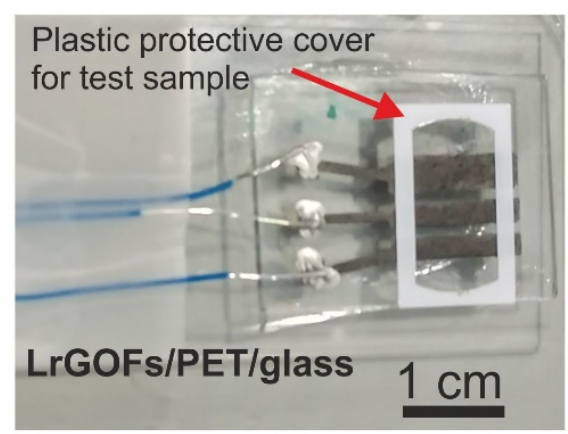

(e)

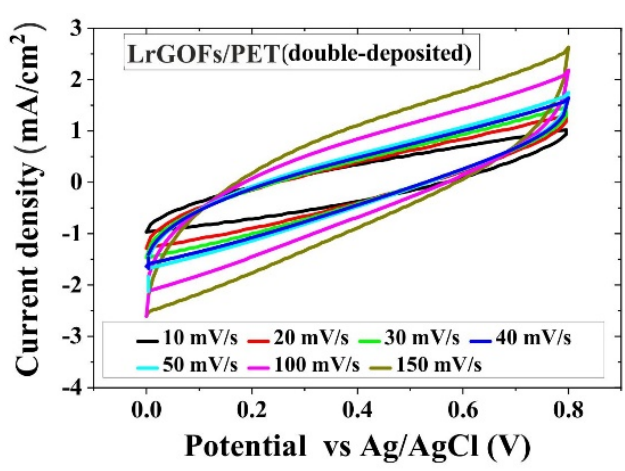

(b)

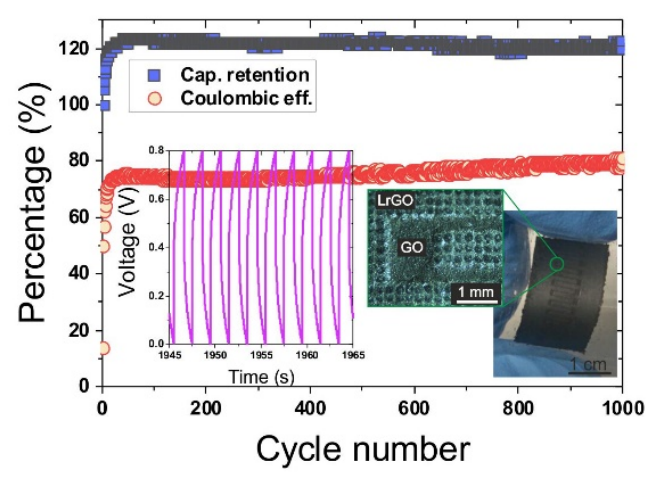

(d)

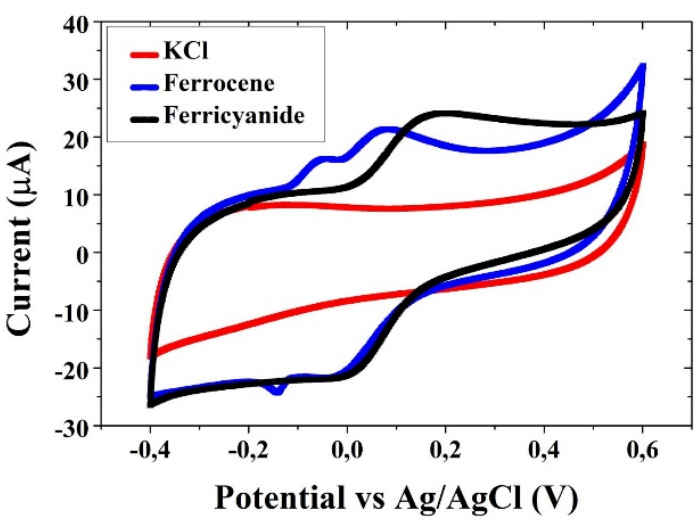

(f)

Figure 8. Cyclic voltammetry plots of (a) single-deposited and (b) double-deposited LrGOF/PET electrodes obtained with $0.25 \mathrm{~mm}$ spot diameter, and scan speed of 10 $\mathrm{mm} / \mathrm{min}$ (double-deposited) and $100 \mathrm{~mm} / \mathrm{min}$ (single-deposited). (d) Capacitance retention, coulombic efficiency and GCD plot showing cycles (inset) of a microsupercapacitor (single-deposited LrGOF/PET) obtained by pixelated mode with $0.25 \mathrm{~mm}$ spot, pulse width of $0.1 \mathrm{~s}$ and $0.2 \mathrm{~mm}$ of separation between spots. The applied current density was $0.9 \mathrm{~mA} / \mathrm{cm}^{2}$. (e) Three-electrode single-deposited LrGOFs/PET electrochemical cell obtained in scanning mode with a $0.25 \mathrm{~mm}$ laser spot and 100 $\mathrm{mm} / \mathrm{min}$ scan speed. (f) Cyclic voltammograms recorded in $0.1 \mathrm{M} \mathrm{KCl}$ solutions 
containing $1 \mathrm{mM}$ ferrocene methanol or $1 \mathrm{mM}$ ferricyanide redox probes. Sweep rate: 50 $\mathrm{mV} / \mathrm{s}$

In summary, the proposed method consisting on laser irradiation of GOFs and GOMs between glass slides is demonstrated to be an effective way for obtaining functional rGObased electrodes. The obtained electrodes show potential to be used in supercapacitor and electrochemical sensing devices, though their functionality should be further optimized. Fig. 9 displays a scheme of an envisaged fabrication process of LrGOFs/PET flexible electrodes based on roll-to-roll processing fully scalable to the industrial environment. This process is non-toxic, rapid, cost-effective and easy to implement. Besides, no special gas environment is required for avoiding oxidation of GO and relatively thick films can be chemically transformed.

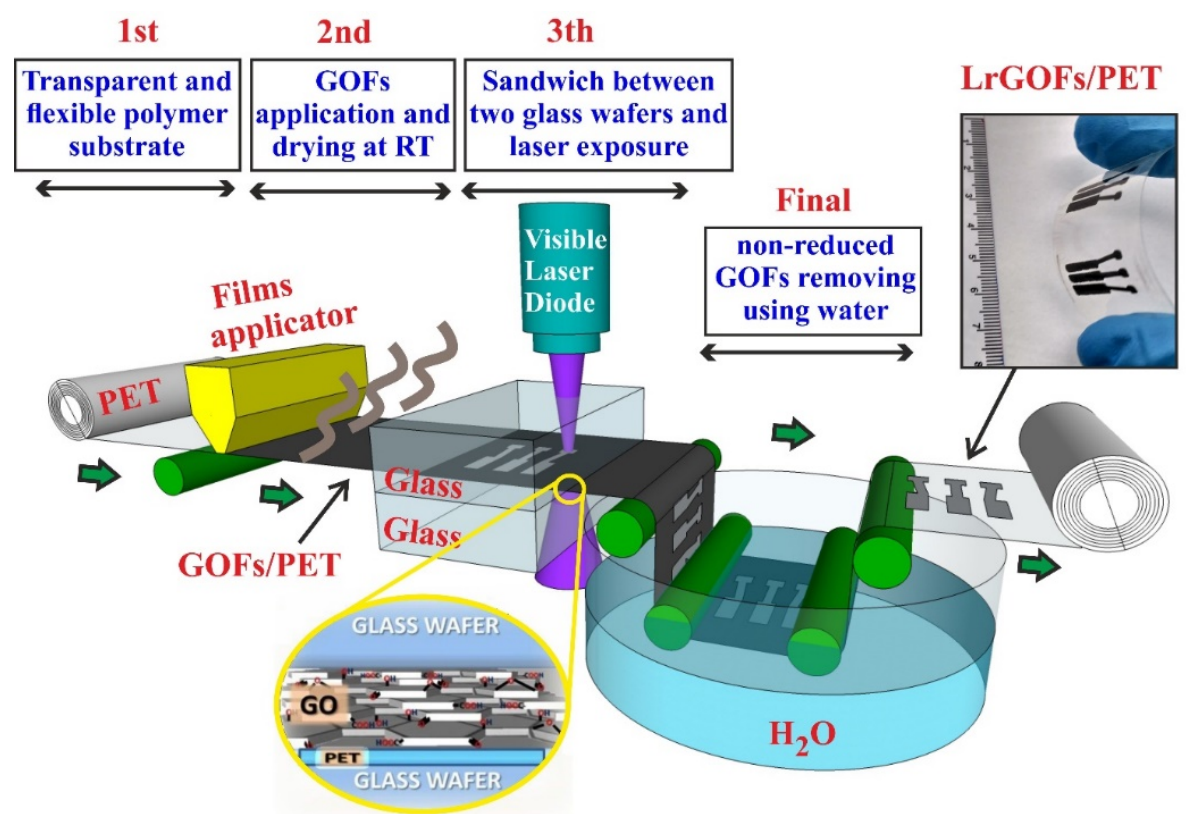

Figure 9. Scheme of a scalable method based on roll-to-roll processing for fabrication of rGO flexible electrodes (LrGOFs/PET).

\section{Conclussions}

A simple and versatile method was envisaged for obtaining reduced graphene oxidebased flexible electrodes either on polymer substrates or as stable self-standing membranes. Neither special environment nor expensive equipment is needed for obtaining relatively thick electrodes with good electrical conductance. The use of 
continuous wave laser radiation allows the tuning of the irradiation time necessary for heating up to the desired depth, and inducing the thermal reduction of the whole GO layer thickness. Moreover, the partial melting of the layer / polymer interphase allows good adherence, which is one of the key problems of the electrodes obtained from graphene inks by means of conventional methods. This technology is fully able to be implemented at the industrial enviroment. The use of higher power laser systems would allow faster manufacturing rates, leading to more competitive throughput. The non-irradiated material can be collected from water immersion and re-deposited as new GOFs.

\section{Acknowledgements}

The authors thank the financial support of the Spanish Ministry of Economy, Industry and Competitiveness under the project ENE2017-89210-C2-1-R, and support from AGAUR of Generalitat de Catalunya through projects 2017 SGR 1086 and 2017 SGR 1771. ICMAB acknowledges financial support from the Spanish Ministry of Economy and Competitiveness, through the "Severo Ochoa" Programme for Centres of Excellence in R\&D (SEV- 2015-0496). Also, this work was supported by a grant of the Romanian Ministry of Research and Innovation, CCCDI - UEFISCDI, project number PN-III-P11.2-PCCDI-2017-0350 / 01.03.2018 (Graphene4Life), within PNCDI III.

\section{References}

[1] Z. Xie, R. Avila, Y. Huang, J.A. Rogers, Flexible and Stretchable Antennas for Biointegrated Electronics, Advanced Materials (2019) 1902767.

[2] H.-L. Park, Y. Lee, N. Kim, D.-G. Seo, G.-T. Go, T.-W. Lee, Flexible Neuromorphic Electronics for Computing, Soft Robotics, and Neuroprosthetics, Advanced Materials (2019) 1903558.

[3] K. Dong, X. Peng, Z.L. Wang, Fiber/Fabric-Based Piezoelectric and Triboelectric Nanogenerators for Flexible/Stretchable and Wearable Electronics and Artificial Intelligence, Advanced Materials (2019) 1902549.

[4] D. Song, A. Mahajan, E.B. Secor, M.C. Hersam, L.F. Francis, C.D. Frisbie, High-Resolution Transfer Printing of Graphene Lines for Fully Printed, Flexible Electronics, Acs Nano 11(7) (2017) 7431-7439.

[5] Y. Yue, Z. Yang, N. Liu, W. Liu, H. Zhang, Y. Ma, C. Yang, J. Su, L. Li, F. Long, Z. Zou, Y. Gao, A Flexible Integrated System Containing a Microsupercapacitor, a Photodetector, and a Wireless Charging Coil, Acs Nano 10(12) (2016) 11249-11257.

[6] J.W. Park, B.H. Kang, H.J. Kim, A Review of Low-Temperature Solution-Processed Metal Oxide Thin-Film Transistors for Flexible Electronics, Advanced Functional Materials (2019) 1904632.

[7] L. Nayak, S. Mohanty, S.K. Nayak, A. Ramadoss, A review on inkjet printing of nanoparticle inks for flexible electronics, Journal of Materials Chemistry C 7(29) (2019) 8771-8795. 
[8] J. Bian, L. Zhou, X. Wan, C. Zhu, B. Yang, Y. Huang, Laser Transfer, Printing, and Assembly Techniques for Flexible Electronics, Advanced Electronic Materials 5(7) (2019) 1800900.

[9] B. Kang, S. Han, J. Kim, S. Ko, M. Yang, One-Step Fabrication of Copper Electrode by LaserInduced Direct Local Reduction and Agglomeration of Copper Oxide Nanoparticle, Journal of Physical Chemistry C 115(48) (2011) 23664-23670.

[10] G.-W. Huang, Q.-P. Feng, H.-M. Xiao, N. Li, S.-Y. Fu, Rapid Laser Printing of Paper-Based Multilayer Circuits, Acs Nano 10(9) (2016) 8895-8903.

[11] D. Bäuerle, Laser Processing and Chemistry Springer, Berlin, 2000.

[12] H. Palneedi, J.H. Park, D. Maurya, M. Peddigari, G.-T. Hwang, V. Annapureddy, J.-W. Kim, J.J. Choi, B.-D. Hahn, S. Priya, K.J. Lee, J. Ryu, Laser Irradiation of Metal Oxide Films and Nanostructures: Applications and Advances, Advanced Materials 30(14) (2018) 1705148.

[13] D.S. Saidina, N. Eawwiboonthanakit, M. Mariatti, S. Fontana, C. Herold, Recent Development of Graphene-Based Ink and Other Conductive Material-Based Inks for Flexible Electronics, Journal of Electronic Materials 48(6) (2019) 3428-3450.

[14] M.R. Ramli, S. Ibrahim, Z. Ahmad, I.S.Z. Abidin, M.F. Ain, Stretchable Conductive Ink Based on Polysiloxane-Silver Composite and Its Application as a Frequency Reconfigurable Patch Antenna for Wearable Electronics, Acs Applied Materials \& Interfaces 11(31) (2019) 2803328042.

[15] V.K.R. Rao, V.K. Abhinav, P.S. Karthik, S.P. Singh, Conductive silver inks and their applications in printed and flexible electronics, Rsc Advances 5(95) (2015) 77760-77790.

[16] H. Sirringhaus, T. Kawase, R.H. Friend, T. Shimoda, M. Inbasekaran, W. Wu, E.P. Woo, Highresolution inkjet printing of all-polymer transistor circuits, Science 290(5499) (2000) 2123-2126. [17] S. Yan, G. Zhang, H. Jiang, F. Li, L. Zhang, Y. Xia, Z. Wang, Y. Wu, H. Li, Highly Stretchable Room-Temperature Self-Healing Conductors Based on Wrinkled Graphene Films for Flexible Electronics, Acs Applied Materials \& Interfaces 11(11) (2019) 10736-10744.

[18] P. He, J. Cao, H. Ding, C. Liu, J. Neilson, Z. Li, I.A. Kinloch, B. Derby, Screen-Printing of a Highly Conductive Graphene Ink for Flexible Printed Electronics, Acs Applied Materials \& Interfaces 11(35) (2019) 32225-32234.

[19] T. Tuan Sang, N.K. Dutta, N.R. Choudhury, Graphene inks for printed flexible electronics: Graphene dispersions, ink formulations, printing techniques and applications, Advances in Colloid and Interface Science 261 (2018) 41-61.

[20] D.R. Dreyer, S. Park, C.W. Bielawski, R.S. Ruoff, The chemistry of graphene oxide, Chemical Society Reviews 39(1) (2010) 228-240.

[21] S. Stankovich, D.A. Dikin, R.D. Piner, K.A. Kohlhaas, A. Kleinhammes, Y. Jia, Y. Wu, S.T. Nguyen, R.S. Ruoff, Synthesis of graphene-based nanosheets via chemical reduction of exfoliated graphite oxide, Carbon 45(7) (2007) 1558-1565.

[22] Z. Fan, K. Wang, T. Wei, J. Yan, L. Song, B. Shao, An environmentally friendly and efficient route for the reduction of graphene oxide by aluminum powder, Carbon 48 (2010) 1686-1689.

[23] I. K. Moon, J. Lee, R. S. Ruoff, H. Lee, Reduced graphene oxide by chemical graphitization, Nature comm. 1 (2010) 73.

[24] A. M. Abdelkader, D. J. Fray, Controlled electrochemical doping of graphene-based 3D nanoarchitecture electrodes for supercapacitors and capacitive deionisation, Nanoscale 9 (2017) 14548.

[25] M. S. Zoromba, M. H. Abdel-Aziz, M. Bassyouni, S. Gutub, D. Demko, A. Abdelkader, Electrochemical Activation of Graphene at Low Temperature: The Synthesis of ThreeDimensional Nanoarchitectures for High Performance Supercapacitors and Capacitive Deionization, ACS Sustainable Chem. Eng. 5, 6 (2017) 4573-4581.

[26] R. Larciprete, S. Fabris, T. Sun, P. Lacovig, A. Baraldi, S. Lizzit, Dual Path Mechanism in the Thermal Reduction of Graphene Oxide, Journal of the American Chemical Society 133(43) (2011) 17315-21. 
[27] A. Perez del Pino, E. Gyorgy, C. Logofatu, J. Puigmarti-Luis, W. Gao, Laser-induced chemical transformation of graphene oxide-iron oxide nanoparticles composites deposited on polymer substrates, Carbon 93 (2015) 373-383.

[28] J. S. Oh, S.-H. Kim, T. Hwang, H.-Y. Kwon, T.H. Lee, A.-H. Bae, H.R. Choi, J.-D. Nam, LaserAssisted Simultaneous Patterning and Transferring of Graphene, Journal of Physical Chemistry C 117(1) (2013) 663-668.

[29] R.D. Rodriguez, G.V. Murastov, A. Lipovka, M.I. Fatkullin, O. Nozdrina, S.K. Pavlov, P.S. Postnikov, M.M. Chehimi, J.-J. Chen, E. Sheremet, High-power laser-patterning graphene oxide: A new approach to making arbitrarily-shaped self-aligned electrodes, Carbon 151 (2019) 148155.

[30] M. F. El-Kady, R. B. Kaner, Scalable fabrication of high-power graphene microsupercapacitors for flexible and on-chip energy storage, Science 335 (2012) 1326-1330.

[31] M. F. El-Kady, M. Ihns, M. Li, J. Y. Hwang, M. F. Mousavi, L. Chaney, A. T. Lech, R. B. Kaner, Engineering three-dimensional hybrid supercapacitors and microsupercapacitors for highperformance integrated energy storage, PNAS 112 (2015) 4233-4238.

[32] M. F. El-Kady, V. Strong, S. Dubin, R. B. Kaner, Laser Scribing of High-Performance and Flexible Graphene-Based Electrochemical Capacitors, Science 335 (2012) 1326.

[33] F.J. Romero, A. Toral-Lopez, A. Ohata, D.P. Morales, F.G. Ruiz, A. Godoy, N. Rodriguez, LaserFabricated Reduced Graphene Oxide Memristors, Nanomaterials 9(6) (2019) 897.

[34] T. Kavinkumar, L.R. Shobin, S. Manivannan, Effect of laser irradiation on electrical and gas sensing properties of reduced graphene oxide-graphene oxide heterostructure films, Journal of Alloys and Compounds 784 (2019) 301-312.

[35] Z. Chu, Y. Wang, L. Jiao, X. Zhang, Laser-scribed reduced graphene oxide as counter electrode for dye-sensitized solar cell, Fullerenes Nanotubes and Carbon Nanostructures (2019). [36] T. Kavinkurnar, P. Kavitha, N. Naresh, S. Manivannan, M. Muneeswaran, B. Neppolian, High performance flexible solid-state symmetric supercapacitors based on laser induced porous reduced graphene oxide-graphene oxide hybrid nanostructure devices, Applied Surface Science 480 (2019) 671-679.

[37] T. Tung Xuan, H. Choi, C. Cuong Huu, J.H. Sul, I.G. Kim, S.-M. Lee, J.-H. Kim, J.B. In, LaserInduced Reduction of Graphene Oxide by Intensity-Modulated Line Beam for Supercapacitor Applications, Acs Applied Materials \& Interfaces 10(46) (2018) 39777-39784.

[38] J. Cai, C. Lv, E. Aoyagi, S. Ogawa, A. Watanabe, Laser Direct Writing of a High-Performance All-Graphene Humidity Sensor Working in a Novel Sensing Mode for Portable Electronics, Acs Applied Materials \& Interfaces 10(28) (2018) 23987-23996.

[39] J. Tang, X. Zhong, H. Li, Y. Li, F. Pan, B. Xu, In-situ and selectively laser reduced graphene oxide sheets as excellent conductive additive for high rate capability LiFePO4 lithium ion batteries, Journal of Power Sources 412 (2019) 677-682.

[40] L. Guo, Y.-W. Hao, P. Yang, P.-L. Li, N. Sun, X.-W. Feng, J. Zhao, C.-A. Chen, J.-F. Song, Fast fabrication of graphene oxide/reduced graphene oxide hybrid hydrogels for thermosensitive smart actuator utilizing laser irradiation, Materials Letters 237 (2019) 245-248.

[41] F.-H. Jin, Y. Pang, W.-L. Cai, J.-M. Jian, L.-L. Ren, Y.-Y. Fan, M.-R. Li, W.-S. Zhao, Y. Yang, S.-F. Yang, T.-L. Ren, High performance and low-cost graphene vacuum pressure sensor based on one-step laser scribing, Applied Physics Letters 114(8) (2019) 081602.

[42] H.H. Shi, S. Jang, H.E. Naguib, Freestanding Laser-Assisted Reduced Graphene Oxide Microribbon Textile Electrode Fabricated on a Liquid Surface for Supercapacitors and Breath Sensors, Acs Applied Materials \& Interfaces 11(30) (2019) 27183-27191.

[43] M. Moussa, S.A. Al-Bataineh, D. Losic, D.P. Dubai, Engineering of high-performance potassium-ion capacitors using polyaniline-derived $\mathrm{N}$-doped carbon nanotubes anode and laser scribed graphene oxide cathode, Applied Materials Today 16 (2019) 425-434.

[44] A.P.d. Pino, A. Chuquitarqui, L.C. Cotet, Procedure for obtaining a flexible electrode (WO 2019/234284 A1), Spain, 2019. 
[45] A.P. del Pino, E. Gyorgy, C. Cotet, L. Baia, C. Logofatu, Laser-induced chemical transformation of free-standing graphene oxide membranes in liquid and gas ammonia environments, Rsc Advances 6(55) (2016) 50034-50042.

[46] L.C. Cotet, K. Magyari, M. Todea, M.C. Dudescu, V. Danciu, L. Baia, Versatile self-assembled graphene oxide membranes obtained under ambient conditions by using a water-ethanol suspension, Journal of Materials Chemistry A 5(5) (2017) 2132-2142.

[47] A. Perez del Pino, A. Martinez Villarroya, A. Chuquitarqui, C. Logofatu, D. Tonti, E. Gyorgy, Reactive laser synthesis of nitrogen-doped hybrid graphene-based electrodes for energy storage, Journal of Materials Chemistry A 6(33) (2018) 16074-16086.

[48] C.-M. Chen, J.-Q. Huang, Q. Zhang, W.-Z. Gong, Q.-H. Yang, M.-Z. Wang, Y.-G. Yang, Annealing a graphene oxide film to produce a free standing high conductive graphene film, Carbon 50(2) (2012) 659-667.

[49] X. Chen, D. Meng, B. Wang, B.-W. Li, W. Li, C.W. Bielawski, R.S. Ruoff, Rapid thermal decomposition of confined graphene oxide films in air, Carbon 101 (2016) 71-76.

[50] S.E. A.M. Dimiev, Graphene Oxide: Fundamentals and Applications, Wiley, 2016.

[51] H. Guo, M. Peng, Z. Zhu, L. Sun, Preparation of reduced graphene oxide by infrared irradiation induced photothermal reduction, Nanoscale 5(19) (2013) 9040-9048.

[52] M.T.H. Aunkor, I.M. Mahbubul, R. Saidur, H.S.C. Metselaar, The green reduction of graphene oxide, Rsc Advances 6(33) (2016) 27807-27828.

[53] A.C. Ferrari, D.M. Basko, Raman spectroscopy as a versatile tool for studying the properties of graphene, Nature Nanotechnology 8(4) (2013) 235-246.

[54] J. B. Wu, M. L. Lin, X. Cong, H. N. Liu, Ping-Heng Tan, Raman spectroscopy of graphenebased materials and its applications in related devices, Chem. Soc. Rev. 47 (2018) 1822-1873.

[55] D. López-Díaz, M. López Holgado, J. L. García-Fierro, M. M. Velázquez, Evolution of the Raman Spectrum with the Chemical Composition of Graphene Oxide, J. Phys. Chem. C 121 (2017) 20489-20497.

[56] S. Pei, H.-M. Cheng, The reduction of graphene oxide, Carbon 50(9) (2012) 3210-3228.

[57] A. Eckmann, A. Felten, A. Mishchenko, L, Britnell, R. Krupke, K. S. Novoselov, C. Casiraghi, Probing the Nature of Defects in Graphene by Raman Spectroscopy, Nano Lett. 12 (2012) 3925-3930.

[58] L. Vicarelli, S.J. Heerema, C. Dekker, H.W. Zandbergen, Controlling Defects in Graphene for Optimizing the Electrical Properties of Graphene Nanodevices, Acs Nano 9(4) (2015) 3428-3435. [59] J. Yuan, L.-P. Ma, S. Pei, J. Du, Y. Su, W. Ren, H.-M. Cheng, Tuning the Electrical and Optical Properties of Graphene by Ozone Treatment for Patterning Monolithic Transparent Electrodes, Acs Nano 7(5) (2013) 4233-4241.

[60] C.-R. Yang, S.-F. Tseng, Y.-T. Chen, Laser-induced reduction of graphene oxide powders by high pulsed ultraviolet laser irradiations, Applied Surface Science 444 (2018) 578-583.

[61] K. Griffiths, C. Dale, J. Hedley, M.D. Kowal, R.B. Kaner, N. Keegan, Laser-scribed graphene presents an opportunity to print a new generation of disposable electrochemical sensors, Nanoscale 6(22) (2014) 13613-13622.

[62] A. Longo, R. Verucchi, L. Aversa, R. Tatti, A. Ambrosio, E. Orabona, U. Coscia, G. Carotenuto, P. Maddalena, Graphene oxide prepared by graphene nanoplatelets and reduced by laser treatment, Nanotechnology 28(22) (2017) 224002.

[63]M. Zhong, F. Zhang, Y. Yu, J. Zhang, W. Shen, S. Guo, Flexible micro-supercapacitors assembled via chemically reduced graphene oxide films assisted by a laser printer, Nanotechnology 29(43) (2018).

[64] K. Li, J. Zhang, Recent advances in flexible supercapacitors based on carbon nanotubes and graphene, Sci China Mater 61(2) (2018) 210-232.

[65] Y. Shao, M. F. El-Kady, L. J. Wang, Q. Zhang, Y. Li, H. Wang, M. F. Mousaviae, R. B. Kaner, Graphene-based materials for flexible supercapacitors, Chem. Soc. Rev. 44 (2015) 3639.

[66] W. Gao, N. Singh, L. Song, Z. Liu, A. L. M. Reddy, L. Ci, R. Vajtai, Q. Zhang, B. Wei, P. M. Ajayan, Direct laser writing of micro-supercapacitors on hydrated graphite oxide films, Nat. 
Nanotechnol. 6 (2011) 496-500.

[67] J. Lin, Z. Peng, Y. Liu, F. Ruiz-Zepeda, R. Ye, E. L. G. Samuel, M. J. Yacaman, B. I. Yakobson, J. M. Tour, Laser-induced porous graphene films from commercial polymers, Nature Comm. 5 (2014) 4714.

[68] J. Vatamanu, M. Vatamanu, D. Bedrov, Non-Faradaic Energy Storage by Room Temperature Ionic Liquids in Nanoporous Electrodes, ACS Nano 9 (2015) 5999-6017.

[69] J. Chmiola, C. Largeot, P.-L. Taberna, P. Simon, Y. Gogotsi, Monolithic carbide derived carbon films for micro-supercapacitors, Science 328 (5977) (2010) 480-483.

[70] P. Niu, L. Asturias-Arribas, X. jorda, A.R. Goni, A. Roig, M. Gich, C. Fernandez-Sanchez, Carbon-Silica Composites to Produce Highly Robust Thin-Film Electrochemical Microdevices, Advanced Materials Technologies 2(12) (2017) 1700163.

[71] R. L. McCreery, Advanced carbon electrode materials for molecular electrochemistry, Chemical Reviews 108 (2008) 2646-2687. 